\title{
ADAPTIVE IMPEDANCE CONTROL OF ROBOT MANIPULATORS WITH PARAMETRIC UNCERTAINTY FOR CONSTRAINED PATH-TRACKING
}

\author{
Isela BONILlA ${ }^{a}$, MARCO MENDOZA $^{a, *}$, DANIEL U. CAMPOS-DELGADO ${ }^{a}$, \\ DIANA E. HERNÁNDEZ-ALFARO ${ }^{b}$
}

\author{
${ }^{a}$ Faculty of Science \\ Autonomous University of San Luis Potosí, Av. Salvador Nava S/N, San Luis Potosí, SLP, 78290 Mexico \\ e-mail: \{isela.bonilla, marco.mendoza\}@uaslp.mx, ducd@fciencias.uaslp.mx \\ ${ }^{b}$ Ericsson \\ Av. 5 de Febrero 1351, Edificio Fresno, Zona Industrial Benito Juárez, Santiago de Querétaro, Qro., 76120 Mexico \\ e-mail: danaeli20@hotmail.com
}

\begin{abstract}
The main impedance control schemes in the task space require accurate knowledge of the kinematics and dynamics of the robotic system to be controlled. In order to eliminate this dependence and preserve the structure of this kind of algorithms, this paper presents an adaptive impedance control approach to robot manipulators with kinematic and dynamic parametric uncertainty. The proposed scheme is an inverse dynamics control law that leads to the closed-loop system having a PD structure whose equilibrium point converges asymptotically to zero according to the formal stability analysis in the Lyapunov sense. In addition, the general structure of the scheme is composed of continuous functions and includes the modeling of most of the physical phenomena present in the dynamics of the robotic system. The main feature of this control scheme is that it allows precise path tracking in both free and constrained spaces (if the robot is in contact with the environment). The proper behavior of the closed-loop system is validated using a two degree-of-freedom robotic arm. For this benchmark good results were obtained and the control objective was achieved despite neglecting non modeled dynamics, such as viscous and Coulomb friction.
\end{abstract}

Keywords: adaptive control, constrained motion, mechanical impedance, robot manipulator.

\section{Introduction}

Nowadays, the range of applications for robotic systems is quite broad and the vast majority are tasks including the intervention of humans in contact with them (Dulęba and Opałka, 2013; Yarza et al., 2013; Martínez et al., 2016; Belter et al., 2016; Rodríguez-Liñán et al., 2017). Medicine is an area that has benefited from the use of robots in help and assistance for the elderly people, rehabilitation therapies, surgeries, etc. (Hagn et al., 2008; Marchal-Crespo and Reinkensmeyer, 2009; Xu et al., 2011; Gribovskaya et al., 2011; Sharifi et al., 2012; Hussain et al., 2013; Pérez-Ibarra et al., 2014; Sharifi et al., 2014; Song et al., 2015; Li et al., 2017). In this kind of applications, safety is an important factor due to the unstructured nature of human-robot interaction tasks, and then suitable control algorithms are required to regulate a

* Corresponding author compliant and stable behavior of the robot.

Several impedance-control algorithms have been proposed by various researchers and demonstrated that can deal with the environmental constraints by considering the relationship between the robot and the environment, characterized by the mechanical impedance and admittance concepts (Hogan, 1985; Anderson and Spong, 1988; Carelli and Kelly, 1991; Chiaverini et al., 1999; Kang et al., 2009; Mendoza et al., 2012). The main limitation of most impedance control schemes is the need for precise knowledge of the parameters and the dynamic model of the robotic system to control. That is why some adaptive/robust impedance controllers have been developed (Carelli and Kelly, 1991; Lu and Meng, 1991; Chien and Huang, 2004; Jiang, 2005; Jianbin et al., 2009; Kang et al., 2009; Xu et al., 2011; Gribovskaya et al., 2011; Sharifi et al., 2012; 2014; Hussain et al., 2013; Pérez-Ibarra et al., 2014, Li et al., 2017; He et al., 2016). 
The classical approach to impedance control was proposed by Hogan (1985), and then its adaptive version was developed for stable execution of contact tasks and preventing a performance degradation due to parameter uncertainties (Lu and Meng, 1991). Having the same goal in mind, a computed torque controller with a parameter estimator was presented by Carelli and Kelly (1991). It was based on a singular system model representation capable of achieving global tracking for constrained motion control. Another technique used to represent the uncertainties is function approximation which was successfully implemented for adaptive impedance control by Chien and Huang (2004). For flexible robots, an adaptive impedance controller was designed by Jiang (2005) based on end-effector trajectory tracking by achieving convergence to a desired manifold, which represents a desirable performance for the system during interaction. In order to restrict contact forces, a collision detection system with joint torque sensors was proposed by Jianbin et al. (2009) by generating an adaptive Cartesian impedance control for flexible joint manipulators.

In the work of Haninger et al. (2016), a disturbance observer based robust control architecture is presented, but only the effect of actuation dynamics is analyzed on a series-elastic actuated system, and the results are not extended to the complete dynamics of a robot manipulator system. The dilemma between impedance accuracy and robustness against modeling errors was successfully addressed by Kang et al. (2009), by using an internal model control approach with time-delay estimation. Recently, He et al. (2016) developed a neural-network based adaptive impedance control to solve the robot-environment interaction problem, which does not require knowledge of robotic dynamics; however, only the convergence of the impedance error to a small neighborhood around zero is ensured and the presented results are limited to the behavior of the robot in joint space. In all these works, the proposed schemes have only attempted to cope with the dynamic uncertainty. Therefore, in this paper we propose an accurate control tool for unconstrained and constrained path tracking under kinematic and dynamic parameter uncertainty. Our adaptive impedance control approach is a result of combining the main ideas presented by Mendoza et al. (2012) as well as Wang and Xie (2009), and it ensures convergence of the impedance error towards zero.

Teleoperation is one of the most common applications of impedance control; for example, in the works of Rahimifard et al. (2016) and Yang et al. (2016), adaptive schemes are used to cope with uncertain nonlinearities in the impedance model and the effect of the unknown payload, respectively. The control scheme proposed by Rahimifard et al. (2016) considers full nonlinear dynamics having uncertainties in the model and the passivity condition is relaxed via a Lyapunov function to show the stability of the closed-loop system in constrained motion. On the other hand, in the work of Yang et al. (2016), a neural learning based compensation scheme is used to overcome the effect of the unknown payload as well as uncertainties associated with the robot model and the environment. However, in both cases, they only try to guarantee the correct path tracking and do not address the problem of robot-environment interaction in detail or the presence of uncertainty in kinematic parameters that affect the performance of this type of tasks in the Cartesian space. Therefore, the proposed control structure will guarantee good results for both free and restricted movements in the task space.

This kind of adaptive scheme represents a useful tool for human-robot interaction, in the context of rehabilitation therapies assisted by robots, a future application of great interest to all of us. Recently, an adaptive impedance controller based on an evolutionary dynamic fuzzy neural network was used to regulate the desired impedance between a robot (therapist) and an impaired limb (patient) for physical recovery (Xu et al., 2011). This adaptive scheme can deal with an uncertain change in the impaired limb's physical condition; the main limitation of this scheme is that it requires an off-line optimization process, as well as an on-line readjustment process. In addition, it presents a discontinuous behavior during force tracking and its theoretical foundation is only supported by an analysis of the convergence of the control method that does not take into account the complete system dynamics.

Alternatively, model reference adaptive controllers have been implemented on rehabilitation robots to compensate model uncertainties such as friction coefficients by improving the tracking performance (Sharifi et al., 2012; 2014). In order to approximate the unknown dead-zone effects in the joints and the robot's dynamics, for an upper limb robotic exoskeleton, an adaptive neural network controller was developed by Li et al. (2017); however, the convergence analysis of tracking errors only ensures that they remain bounded and the validation results are limited to show the robot behavior in joint space. For lower limb rehabilitation, some adaptive schemes have also been proposed (Hussain et al., 2013; Pérez-Ibarra et al., 2014). For example, assist-as-needed gait training is addressed with adaptive impedance control and it adapts the robotic assistance according to the disability level and voluntary participation of the users (Hussain et al., 2013). The adaptive control approach presented herein could be used in robotic systems for rehabilitation, because its ability to precisely track trajectories, in free and restricted space, is a desirable feature for passive and active therapy sessions.

Note that the proposed control approach allows us to lead the closed-loop system to an asymptotically-stable 
equilibrium point and the corresponding analysis, by Lyapunov's direct method, is presented. In addition, computer simulations using the model of a direct-drive robot manipulator of two degrees of freedom, corroborate the suitable performance of the proposed control scheme for constrained path tracking.

\section{Preliminaries}

2.1. Notation. Let $\boldsymbol{A} \in \mathbb{R}^{m \times n}$ and $\boldsymbol{y} \in \mathbb{R}^{n}$. As usual, $a_{i j}$ represents the element of $\boldsymbol{A}$ in the $i$-th row and $j$-th column, and $y_{i}$ is the $i^{t h}$ element of $\boldsymbol{y} . \mathbf{0}_{n}$ represents the origin of $\mathbb{R}^{n}, \boldsymbol{I}_{n}$ denotes the $n \times n$ identity matrix. Throughout this work, $\|\cdot\|$ represents the standard Euclidean norm for vectors, i.e.,

$$
\|\boldsymbol{y}\|=\sqrt{\boldsymbol{y}^{T} \boldsymbol{y}}=\sqrt{\sum_{i=1}^{n} y_{i}^{2}},
$$

and the induced norm for matrices, i.e.,

$$
\|\boldsymbol{A}\|=\sqrt{\lambda_{\max }\left\{\boldsymbol{A}^{T} \boldsymbol{A}\right\}} .
$$

Let $\mathcal{L}_{2}^{n}$ be the set of continuous functions $l: \mathbb{R}_{+} \rightarrow$ $\mathbb{R}^{n}$ such that

$$
\int_{0}^{\infty} \boldsymbol{l}^{T}(t) \boldsymbol{l}(t) \mathrm{d} t=\int_{0}^{\infty}\|\boldsymbol{l}(t)\|^{2} \mathrm{~d} t<\infty .
$$

Then a function $\boldsymbol{l} \in \mathcal{L}_{2}^{n}$ if its energy is bounded, i.e., $\boldsymbol{l}$ is square integrable. On the other hand, the $\mathcal{L}_{\infty}^{n}$ space consists of the set of continuous functions $l: \mathbb{R}_{+} \rightarrow \mathbb{R}^{n}$ such that

$$
\sup _{t \geq 0}\|\boldsymbol{l}(t)\|<\infty,
$$

i.e., their Euclidean norms are bounded, $\boldsymbol{l} \in \mathcal{L}_{\infty}^{n}$. Therefore, we can conclude that $\boldsymbol{l}$ is bounded and square integrable if $\boldsymbol{l} \in \mathcal{L}_{2}^{n}$.

Consider a locally Lipschitz-continuous scalar function $\phi: \mathbb{R} \mapsto \mathbb{R}$, vanishing at zero, i.e., $\phi(0)=0$. $D^{+} \phi$ denotes the upper-right derivative of $\phi$, i.e.,

$$
D^{+} \phi(\zeta)=\lim \sup _{h \rightarrow 0^{+}} \frac{\phi(\zeta+h)-\phi(\zeta)}{h}
$$

(cf. Khalil, 1996; Rouche et al., 1977).

2.2. Kinematic modeling. Consider a serial rigid robot manipulator with $n$ degrees of freedom, whose direct kinematics mapping $\mathcal{K}: \mathbb{R}^{n} \mapsto \mathbb{R}^{m}$ is given by

$$
\boldsymbol{x}=\mathcal{K}(\boldsymbol{q}),
$$

where $q \in \mathbb{R}^{n}$ represents the joint-space position vector and $\boldsymbol{x} \in \mathbb{R}^{m}$ is the task-space posture vector. The derivatives of (1) with respect to time can be represented as

$$
\begin{aligned}
& \dot{\boldsymbol{x}}=\boldsymbol{J}(\boldsymbol{q}) \dot{\boldsymbol{q}}, \\
& \ddot{\boldsymbol{x}}=\boldsymbol{J}(\boldsymbol{q}) \ddot{\boldsymbol{q}}+\dot{\boldsymbol{J}}(\boldsymbol{q}) \dot{\boldsymbol{q}},
\end{aligned}
$$

where $\dot{\boldsymbol{q}}, \ddot{\boldsymbol{q}} \in \mathbb{R}^{n}$ represent the vectors of joint velocity and acceleration, respectively; $\dot{\boldsymbol{x}}, \ddot{\boldsymbol{x}} \in \mathbb{R}^{m}$ are the vectors of task-space velocity and acceleration, respectively; $\boldsymbol{J}(\boldsymbol{q})=\partial \mathcal{K} / \partial \boldsymbol{q} \in \mathbb{R}^{m \times n}$ represents the analytical Jacobian matrix of the robot manipulator (Sciavicco and Siciliano, 2000), whose time derivative is $\dot{\boldsymbol{J}}(\boldsymbol{q})=$ $\mathrm{d} \boldsymbol{J} / \mathrm{d} t \in \mathbb{R}^{m \times n}$.

Property 1. There exists a set of parameters $\boldsymbol{\theta}_{k}=$ $\left[\theta_{k 1}, \theta_{k 2}, \ldots, \theta_{k r}\right]^{T}$ such that the kinematic relationship in Eqn. (2) can be rewritten as

$$
\dot{\boldsymbol{x}}=\boldsymbol{Y}_{k}(\boldsymbol{q}, \dot{\boldsymbol{q}}) \boldsymbol{\theta}_{k},
$$

where $\boldsymbol{Y}_{k}(\boldsymbol{q}, \dot{\boldsymbol{q}}) \in \mathbb{R}^{m \times r}$ represents a kinematic regression matrix (Wang and Xie, 2009). In order to avoid the need for measuring the Cartesian (task-space) velocity for parameter-estimation purposes, a low-pass filtering of the task-space velocity can be used (Wang and Xie, 2009). Then, with abuse of notation

$$
\boldsymbol{z} \triangleq \frac{\lambda}{\lambda+s} \dot{\boldsymbol{x}}=\boldsymbol{W}_{k} \boldsymbol{\theta}_{k},
$$

where $\lambda>0$ represents the cutoff frequency and $z \in$ $\mathbb{R}^{m}$ is the filtered output with initial condition $\boldsymbol{z}(0)=$ $\mathbf{0}_{m} ; \boldsymbol{W}_{k} \triangleq[\lambda /(\lambda+s)] \boldsymbol{Y}_{k}(\boldsymbol{q}, \dot{\boldsymbol{q}})$ with initial condition $\boldsymbol{W}_{k}(0)=\mathbf{0}_{m \times r}$. Therefore, in practice, $\boldsymbol{z}$ can be implemented as $[\lambda s /(\lambda+s)] \boldsymbol{x}$ by measuring only the task-space position.

2.3. Dynamic modeling. According to the principle of virtual work, in the work of Takegaki and Arimoto (1981) it is stated that

$$
\boldsymbol{\tau}=\boldsymbol{J}^{T}(\boldsymbol{q}) \boldsymbol{f},
$$

where $\tau \in \mathbb{R}^{n}$ represents the generalized joint torques, and $\boldsymbol{f} \in \mathbb{R}^{m}$ is the vector of task-space force and torque components operating at the robot's end-effector. Therefore, the dynamic model of an $n$-degree-of-freedom serial and rigid robot manipulator, interacting (or in contact) with the environment, can be represented (Sciavicco and Siciliano, 2000; Canudas et al., 1996) as

$$
\boldsymbol{M}(\boldsymbol{q}) \ddot{\boldsymbol{q}}+\boldsymbol{C}(\boldsymbol{q}, \dot{\boldsymbol{q}}) \dot{\boldsymbol{q}}+\boldsymbol{g}(\boldsymbol{q})=\boldsymbol{\tau}-\boldsymbol{J}^{T}(\boldsymbol{q}) \boldsymbol{f}_{e},
$$

where $\boldsymbol{M}(\boldsymbol{q}) \in \mathbb{R}^{n \times n}$ is the inertia matrix, $\boldsymbol{C}(\boldsymbol{q}, \dot{\boldsymbol{q}}) \dot{\boldsymbol{q}}$ $\in \mathbb{R}^{n}$ is the vector of Coriolis and centrifugal forces, while $\boldsymbol{g}(\boldsymbol{q})=\nabla \mathcal{U}(\boldsymbol{q}) \in \mathbb{R}^{n}$ is the vector of gravity forces, with $\mathcal{U}(\boldsymbol{q})$ representing the gravitational potential energy. Finally, $\boldsymbol{f}_{e} \in \mathbb{R}^{m}$ is the vector of external contact forces. Some well-known properties characterizing the terms of such a dynamical model are recalled here (see, e.g., Kelly et al., 2005). 
Property 2. $\boldsymbol{M}(\boldsymbol{q})$ is a symmetric, positive definite bounded matrix, i.e., $\mu_{m} \boldsymbol{I}_{n} \leq \boldsymbol{M}(\boldsymbol{q}) \leq \mu_{M} \boldsymbol{I}_{n}, \forall \boldsymbol{q} \in$ $\mathbb{R}^{n}$, for some constants $0<\mu_{m} \leq \mu_{M}$. Then for any position vector $\boldsymbol{q}$, there exists a symmetric, positive definite matrix $\boldsymbol{M}^{-1}(\boldsymbol{q})$ that represents the inverse of $M(q)$

Property 3. For all $\boldsymbol{q}, \dot{\boldsymbol{q}}, \ddot{\boldsymbol{q}} \in \mathbb{R}^{n}$ we have

$$
\boldsymbol{M}(\boldsymbol{q}) \ddot{\boldsymbol{q}}+\boldsymbol{C}(\boldsymbol{q}, \dot{\boldsymbol{q}}) \dot{\boldsymbol{q}}+\boldsymbol{g}(\boldsymbol{q})=\boldsymbol{Y}_{d}(\boldsymbol{q}, \dot{\boldsymbol{q}}, \ddot{\boldsymbol{q}}) \boldsymbol{\theta}_{d},
$$

where $\boldsymbol{Y}_{d}(\boldsymbol{q}, \dot{\boldsymbol{q}}, \ddot{\boldsymbol{q}}) \in \mathbb{R}^{n \times p}$ is a regression matrix and the vector $\boldsymbol{\theta}_{d}=\left[\theta_{d 1}, \theta_{d 2}, \ldots, \theta_{d p}\right]^{T} \in \mathbb{R}^{p}$ depends only on the dynamic parameters of the manipulator. Moreover, if $\boldsymbol{q}, \dot{\boldsymbol{q}}, \ddot{\boldsymbol{q}} \in \mathcal{L}_{\infty}^{n}$ then $\boldsymbol{Y}_{d}(\boldsymbol{q}, \dot{\boldsymbol{q}}, \ddot{\boldsymbol{q}}) \in \mathcal{L}_{\infty}^{n \times p}$

Additionally, the vector of external contact torques $\boldsymbol{J}^{T}(\boldsymbol{q}) \boldsymbol{f}_{e}$ depends only on the vector of kinematic parameters $\boldsymbol{\theta}_{k}$. Therefore, by measuring the contact forces $\boldsymbol{f}_{e}$ the following useful property can be obtained

Property 4. The vector of external contact torques $\boldsymbol{J}^{T}(\boldsymbol{q}) \boldsymbol{f}_{e}$ can be represented as

$$
\boldsymbol{J}^{T}(\boldsymbol{q}) \boldsymbol{f}_{e}=\boldsymbol{Y}_{e}\left(\boldsymbol{q}, \boldsymbol{f}_{e}\right) \boldsymbol{\theta}_{k},
$$

where $\boldsymbol{Y}_{e}\left(\boldsymbol{q}, \boldsymbol{f}_{e}\right) \in \mathbb{R}^{n \times r}$ signifies a regression matrix associated with the robot-environment interaction.

Note that $\boldsymbol{J}(\boldsymbol{q})$ has to be a full-rank matrix for all position vectors $\boldsymbol{q}$ in order to have a singularity-free workspace for the robot manipulator. Thus, the following assumption turns out to be crucial for the subsequent derivations.

\section{Assumption 1. $\operatorname{rank}\{\boldsymbol{J}(\boldsymbol{q})\}=n$.}

\section{Adaptive impedance controller}

In this paper, the problem of impedance control is addressed from the point of view formulated by Mendoza et al. (2012), where the motion control of robot manipulators in task space is generalized for constrained path-tracking. The impedance error $\tilde{\xi} \in \mathbb{R}^{m}$ is then defined as (Carelli and Kelly, 1991)

$$
\tilde{\boldsymbol{\xi}} \triangleq \tilde{\boldsymbol{x}}-\boldsymbol{x}_{f_{e}},
$$

where $\tilde{\boldsymbol{x}}=\boldsymbol{x}_{d}-\boldsymbol{x}$ represents the unconstrained path-tracking error, with $\boldsymbol{x}_{d} \in \mathbb{R}^{m}$ being a desired trajectory; and $\boldsymbol{x}_{f_{e}} \triangleq \mathcal{F}(s) \boldsymbol{f}_{e}$ is the adjustment vector obtained from the filtered external contact force, with

$$
\mathcal{F}(s) \triangleq\left[s^{2} \boldsymbol{M}_{d}+s \boldsymbol{B}_{d}+\boldsymbol{K}_{d}\right]^{-1}
$$

representing the operator related to a second-order multi-dimensional filter with $\boldsymbol{K}_{d}, \boldsymbol{B}_{d}, \boldsymbol{M}_{d} \in$ $\mathbb{R}^{m \times m}$ being diagonal, positive definite matrices of stiffness, damping and inertia, respectively, i.e., $\boldsymbol{K}_{d}=$ $\operatorname{diag}\left[k_{d 1}, \ldots, k_{d m}\right], \boldsymbol{B}_{d}=\operatorname{diag}\left[b_{d 1}, \ldots, b_{d m}\right]$ and $\boldsymbol{M}_{d}=$ $\operatorname{diag}\left[m_{d 1}, \ldots, m_{d m}\right]$ with $k_{d i}>0, b_{d i}>0$ and $m_{d i}>0$ $\forall i=1, \ldots, m$, such that

$$
b_{d i} \geq \sqrt{4 k_{d i} m_{d i}} .
$$

Then $\mathcal{F}(s)$ is a stable, second-order linear filter (Mendoza et al., 2012).

From the definition of impedance error in Eqn. (10), with abuse of notation, the vectors

$$
\begin{aligned}
& \dot{\tilde{\xi}}=\dot{\boldsymbol{x}}_{d}-\dot{\boldsymbol{x}}-\dot{\boldsymbol{x}}_{f_{e}}, \\
& \ddot{\tilde{\boldsymbol{\xi}}}=\ddot{\boldsymbol{x}}_{d}-\ddot{\boldsymbol{x}}-\ddot{\boldsymbol{x}}_{f_{e}}
\end{aligned}
$$

represent the time derivatives of the impedance error.

Formally, the main objective of impedance control consists in selecting $\tau$ in such a way that

$$
\begin{aligned}
& \lim _{t \rightarrow \infty} \tilde{\boldsymbol{\xi}}(t)=\mathbf{0}_{m}, \\
& \lim _{t \rightarrow \infty} \dot{\tilde{\xi}}(t)=\mathbf{0}_{m} .
\end{aligned}
$$

If there is no robot-environment interaction, i.e., $\boldsymbol{f}_{e} \equiv$ $\mathbf{0}_{m}$, the objective of impedance control is equivalent to the objective of unconstrained motion control in the task space.

The proposed adaptive impedance controller, based on the concept of inverse dynamics (Spong et al., 2005; Wang and Xie, 2009), is defined as

$$
\begin{aligned}
\boldsymbol{\tau}= & \hat{\boldsymbol{M}}_{0}(\boldsymbol{q}) \hat{\boldsymbol{J}}^{-1}(\boldsymbol{q})[\boldsymbol{a}-\dot{\hat{\boldsymbol{J}}}(\boldsymbol{q}) \dot{\boldsymbol{q}}]+\hat{\boldsymbol{C}}(\boldsymbol{q}, \dot{\boldsymbol{q}}) \dot{\boldsymbol{q}} \\
& +\hat{\boldsymbol{g}}(\boldsymbol{q})+\hat{\boldsymbol{J}}^{T}(\boldsymbol{q}) \boldsymbol{f}_{e}+\boldsymbol{\delta}_{M},
\end{aligned}
$$

where $\hat{M}_{0}(\boldsymbol{q})$ is a symmetric, positive definite matrix that represents the initial estimate of $\boldsymbol{M}(\boldsymbol{q})$ with $\hat{\boldsymbol{\theta}}_{d 0}$ being the initial estimate of $\boldsymbol{\theta}_{d} ; \hat{\boldsymbol{J}}(\boldsymbol{q}), \hat{\boldsymbol{C}}(\boldsymbol{q}, \dot{\boldsymbol{q}})$ and $\hat{\boldsymbol{g}}(\boldsymbol{q})$ represent the estimates of $\boldsymbol{J}(\boldsymbol{q}), \boldsymbol{C}(\boldsymbol{q}, \dot{\boldsymbol{q}})$ and $\boldsymbol{g}(\boldsymbol{q})$, respectively, with $\hat{\boldsymbol{\theta}}_{k}$ and $\hat{\boldsymbol{\theta}}_{d}$ being the current estimated values of $\boldsymbol{\theta}_{k}$ and $\boldsymbol{\theta}_{d}$, and coming from the following auxiliary dynamics

$$
\begin{aligned}
\dot{\hat{\boldsymbol{\theta}}}_{d}= & \boldsymbol{\Gamma}_{d} \boldsymbol{\Phi}_{d}^{T}(\boldsymbol{q}, \dot{\boldsymbol{q}}, \ddot{\boldsymbol{q}}) \boldsymbol{M}_{d}[\tilde{\boldsymbol{\xi}}+\hat{\boldsymbol{\zeta}}] \\
\dot{\hat{\boldsymbol{\theta}}}_{k}= & \boldsymbol{\Gamma}_{k}\left[\boldsymbol{\Phi}_{e}^{T}\left(\boldsymbol{q}, \boldsymbol{f}_{e}\right) \boldsymbol{M}_{d}[\tilde{\boldsymbol{\xi}}+\hat{\boldsymbol{\zeta}}]\right. \\
& \left.-\boldsymbol{W}_{k}^{T} \boldsymbol{\Lambda}_{k}\left(\boldsymbol{W}_{k} \hat{\boldsymbol{\theta}}_{k}-\boldsymbol{z}\right)\right],
\end{aligned}
$$

where $\boldsymbol{z}=\boldsymbol{W}_{k} \boldsymbol{\theta}_{k}, \boldsymbol{\Gamma}_{d} \in \mathbb{R}^{p \times p}, \boldsymbol{\Gamma}_{k} \in \mathbb{R}^{r \times r}$ and $\boldsymbol{\Lambda}_{k} \in$ $\mathbb{R}^{m \times m}$ are diagonal, positive definite matrices of tuning parameters, $\boldsymbol{\Phi}_{d}(\boldsymbol{q}, \dot{\boldsymbol{q}}, \ddot{\boldsymbol{q}}) \triangleq \hat{\boldsymbol{J}}(\boldsymbol{q}) \hat{\boldsymbol{M}}_{0}^{-1}(\boldsymbol{q}) \boldsymbol{Y}_{d}(\boldsymbol{q}, \dot{\boldsymbol{q}}, \ddot{\boldsymbol{q}})$ and $\boldsymbol{\Phi}_{e}\left(\boldsymbol{q}, \boldsymbol{f}_{e}\right) \triangleq \hat{\boldsymbol{J}}(\boldsymbol{q}) \hat{\boldsymbol{M}}_{0}^{-1}(\boldsymbol{q}) \boldsymbol{Y}_{e}\left(\boldsymbol{q}, \boldsymbol{f}_{e}\right)$. The estimate of $\dot{\tilde{\boldsymbol{\xi}}}$ is represented by $\hat{\boldsymbol{\zeta}} \triangleq \dot{\boldsymbol{x}}_{d}-\hat{\dot{\boldsymbol{x}}}-\dot{\boldsymbol{x}}_{f_{e}}$, since $\hat{\boldsymbol{x}}=\hat{\boldsymbol{J}}(\boldsymbol{q}) \dot{\boldsymbol{q}}$ corresponds to the estimate of $\dot{\boldsymbol{x}}$. Meanwhile,

$$
\boldsymbol{a}=\ddot{\boldsymbol{x}}_{d}-\ddot{\boldsymbol{x}}_{f_{e}}+\boldsymbol{M}_{d}^{-1}\left[\nabla \mathcal{U}_{P}(\tilde{\boldsymbol{\xi}})+\boldsymbol{K}_{D} \hat{\boldsymbol{\zeta}}\right]
$$


where $\boldsymbol{K}_{D} \in \mathbb{R}^{m \times m}$ is a diagonal, definite positive matrix of derivative gains, i.e., $\boldsymbol{K}_{D}=$ $\operatorname{diag}\left[k_{D 1}, \ldots, k_{D m}\right]$ with $k_{D i}>0 \forall i=1, \ldots, m$, such that

$$
\min _{i}\left\{k_{D i}\right\}>\max _{i}\left\{m_{d i}\right\}
$$

and $\nabla \mathcal{U}_{P}(\tilde{\boldsymbol{\xi}}) \in \mathbb{R}^{m}$ represents the gradient of an artificial potential-energy function $\mathcal{U}_{P}: \mathbb{R}^{m} \mapsto \mathbb{R}$ satisfying

$$
\begin{array}{rlrl}
\mathcal{U}_{P}(\tilde{\boldsymbol{\xi}}) & >0, & \forall \tilde{\boldsymbol{\xi}} \neq \mathbf{0}_{m}, \\
\mathcal{U}_{P}\left(\mathbf{0}_{m}\right) & =0, \\
\tilde{\boldsymbol{\xi}}^{T} \nabla \mathcal{U}_{P}(\tilde{\boldsymbol{\xi}}) & >0, \quad \forall \tilde{\boldsymbol{\xi}} \neq \mathbf{0}_{m}, \\
\nabla \mathcal{U}_{P}\left(\mathbf{0}_{m}\right) & =\mathbf{0}_{m} .
\end{array}
$$

Finally, as in the work of Wang and Xie (2009), we define

$$
\boldsymbol{\delta}_{M} \triangleq\left[\hat{\boldsymbol{M}}(\boldsymbol{q})-\hat{\boldsymbol{M}}_{0}(\boldsymbol{q})\right] \ddot{\boldsymbol{q}}
$$

to compensate the differences between $\hat{M}_{0}(\boldsymbol{q})$ and $\boldsymbol{M}(\boldsymbol{q})$.

In order to simplify the notation, in what follows, the arguments of some functions will be omitted.

\section{Stability analysis}

Proposition 1. Consider the robots dynamics in (7) by taking $\tau$ as defined in Eqns. (16)-(19) and (25), with Assumption $\square$ and the conditions on the function $\mathcal{U}_{P}(\tilde{\boldsymbol{\xi}})$ stated through the expressions (21)-(24). Then for any positive definite diagonal matrices $\boldsymbol{\Gamma}_{k}, \boldsymbol{\Gamma}_{d}, \boldsymbol{\Lambda}_{k}, \boldsymbol{K}_{d}, \boldsymbol{B}_{d}$, $\boldsymbol{M}_{d}$ and $\boldsymbol{K}_{D}$ such that inequalities (12) and (20) are satisfied, the convergence of impedance errors is guaranteed, i.e., $\tilde{\boldsymbol{\xi}} \rightarrow \mathbf{0}_{m}$ and $\dot{\tilde{\boldsymbol{\xi}}} \rightarrow \mathbf{0}_{m}$ as $t \rightarrow \infty$.

Proof. Observe that by combining Eqns. (7), (16), (19) and (25), the following dynamical system is obtained:

$$
\begin{array}{r}
{[\boldsymbol{M}-\hat{\boldsymbol{M}}] \ddot{\boldsymbol{q}}+[\boldsymbol{C}-\hat{\boldsymbol{C}}] \dot{\boldsymbol{q}}+[\boldsymbol{g}-\hat{\boldsymbol{g}}]+\left[\boldsymbol{J}^{T}-\hat{\boldsymbol{J}}^{T}\right] \boldsymbol{f}_{e}} \\
=\hat{\boldsymbol{M}}_{0} \hat{\boldsymbol{J}}^{-1}\left[\ddot{\boldsymbol{x}}_{d}-\ddot{\boldsymbol{x}}_{f_{e}}\right. \\
\left.+\boldsymbol{M}_{d}^{-1}\left[\nabla \mathcal{U}_{P}+\boldsymbol{K}_{D} \hat{\boldsymbol{\zeta}}\right]-\dot{\hat{\boldsymbol{J}}} \dot{\boldsymbol{q}}\right]-\hat{\boldsymbol{M}}{ }_{0} \ddot{\boldsymbol{q}} \\
\boldsymbol{Y}_{d} \tilde{\boldsymbol{\theta}}_{d}+\boldsymbol{Y}_{e} \tilde{\boldsymbol{\theta}}_{k}=\hat{\boldsymbol{M}}_{0} \hat{\boldsymbol{J}}^{-1}\left[\ddot{\boldsymbol{x}}_{d}-\ddot{\boldsymbol{x}}_{f_{e}}\right. \\
\left.+\boldsymbol{M}_{d}^{-1}\left[\nabla \mathcal{U}_{P}+\boldsymbol{K}_{D} \hat{\boldsymbol{\zeta}}\right]-\dot{\hat{\boldsymbol{J}}} \dot{\boldsymbol{q}}-\hat{\boldsymbol{J}} \ddot{\boldsymbol{q}}\right], \\
\boldsymbol{\Phi}_{d} \tilde{\boldsymbol{\theta}}_{d}+\boldsymbol{\Phi}_{e} \tilde{\boldsymbol{\theta}}_{k}=\hat{\boldsymbol{\zeta}}+\boldsymbol{M}_{d}^{-1}\left[\nabla \mathcal{U}_{P}+\boldsymbol{K}_{D} \hat{\boldsymbol{\zeta}}\right],
\end{array}
$$

where $\tilde{\boldsymbol{\theta}}_{d}=\boldsymbol{\theta}_{d}-\hat{\boldsymbol{\theta}}_{d}, \tilde{\boldsymbol{\theta}}_{k}=\boldsymbol{\theta}_{k}-\hat{\boldsymbol{\theta}}_{k}$ and

$$
\hat{\dot{\boldsymbol{\zeta}}}=\ddot{\boldsymbol{x}}_{d}-\hat{\ddot{\boldsymbol{x}}}-\ddot{\boldsymbol{x}}_{f_{e}}
$$

with $\hat{\ddot{\boldsymbol{x}}}=\hat{\boldsymbol{J}}(\boldsymbol{q}) \ddot{\boldsymbol{q}}+\dot{\hat{\boldsymbol{J}}}(\boldsymbol{q}) \dot{\boldsymbol{q}}$ being the estimate of $\ddot{\boldsymbol{x}}$. Then the closed-loop dynamics takes the following form:

$$
\begin{aligned}
\hat{\dot{\boldsymbol{\zeta}}} & =\boldsymbol{\Phi}_{d} \tilde{\boldsymbol{\theta}}_{d}+\boldsymbol{\Phi}_{e} \tilde{\boldsymbol{\theta}}_{k}-\boldsymbol{M}_{d}^{-1}\left[\nabla \mathcal{U}_{P}+\boldsymbol{K}_{D} \hat{\boldsymbol{\zeta}}\right] \\
\dot{\tilde{\boldsymbol{\theta}}}_{d} & =-\boldsymbol{\Gamma}_{d} \boldsymbol{\Phi}_{d}^{T} \boldsymbol{M}_{d}[\tilde{\boldsymbol{\xi}}+\hat{\boldsymbol{\zeta}}] \\
\dot{\tilde{\boldsymbol{\theta}}}_{k} & =-\boldsymbol{\Gamma}_{k}\left[\boldsymbol{\Phi}_{e}^{T} \boldsymbol{M}_{d}[\tilde{\boldsymbol{\xi}}+\hat{\boldsymbol{\zeta}}]+\boldsymbol{W}_{k}^{T} \boldsymbol{\Lambda}_{k} \boldsymbol{W}_{k} \tilde{\boldsymbol{\theta}}_{k}\right]
\end{aligned}
$$

Note that, from Eqns. (28) under stationary conditions, i.e., for $\hat{\dot{\boldsymbol{\zeta}}}=\hat{\boldsymbol{\zeta}}=\mathbf{0}_{m}, \dot{\tilde{\boldsymbol{\theta}}}_{d}=\mathbf{0}_{p}$ and $\dot{\tilde{\boldsymbol{\theta}}}_{k}=\mathbf{0}_{r}$, we have that

$$
\begin{aligned}
\boldsymbol{\Phi}_{d} \tilde{\boldsymbol{\theta}}_{d}+\boldsymbol{\Phi}_{e} \tilde{\boldsymbol{\theta}}_{k}-\boldsymbol{M}_{d}^{-1} \nabla \mathcal{U}_{P} & =\mathbf{0}_{m} \\
\boldsymbol{\Phi}_{d}^{T} \boldsymbol{M}_{d} \tilde{\boldsymbol{\xi}} & =\mathbf{0}_{p} \\
\boldsymbol{\Phi}_{e}^{T} \boldsymbol{M}_{d} \tilde{\boldsymbol{\xi}}+\boldsymbol{W}_{k}^{T} \boldsymbol{\Lambda}_{k} \boldsymbol{W}_{k} \tilde{\boldsymbol{\theta}}_{k} & =\mathbf{0}_{r}
\end{aligned}
$$

and, premultiplying $29 \mathrm{a}$ by $\tilde{\boldsymbol{\xi}}^{T} \boldsymbol{M}_{d}$,

$$
\tilde{\boldsymbol{\xi}}^{T} \boldsymbol{M}_{d}\left[\boldsymbol{\Phi}_{d} \tilde{\boldsymbol{\theta}}_{d}+\boldsymbol{\Phi}_{e} \tilde{\boldsymbol{\theta}}_{k}\right]=\tilde{\boldsymbol{\xi}}^{T} \nabla \mathcal{U}_{P}
$$

Thus, by combining (29b), 29c and 2 , we get

$$
\begin{aligned}
\tilde{\boldsymbol{\theta}}_{k}^{T} \boldsymbol{\Phi}_{e}^{T} \boldsymbol{M}_{d} \tilde{\boldsymbol{\xi}} & =\tilde{\boldsymbol{\xi}}^{T} \nabla \mathcal{U}_{P}, \\
\tilde{\boldsymbol{\xi}}^{T} \nabla \mathcal{U}_{P}+\tilde{\boldsymbol{\theta}}_{k}^{T} \boldsymbol{W}_{k}^{T} \boldsymbol{\Lambda}_{k} \boldsymbol{W}_{k} \tilde{\boldsymbol{\theta}}_{k} & =0 .
\end{aligned}
$$

From Assumption 1 and the conditions on the function $\mathcal{U}_{P}(\tilde{\boldsymbol{\xi}})$ stated through the expressions (23)-(24), $\tilde{\boldsymbol{\xi}}=\mathbf{0}_{m}$ and $\tilde{\boldsymbol{\theta}}_{k}=\mathbf{0}_{r}$ are the equilibrium conditions, while the equilibrium condition for the estimation error $\tilde{\boldsymbol{\theta}}_{d}$ turns out to be defined by the solutions of the equation $\boldsymbol{\Phi}_{d} \tilde{\boldsymbol{\theta}}_{d}=\mathbf{0}_{m}$. Consequently, $\tilde{\boldsymbol{\theta}}_{d} \in \operatorname{ker}\left(\boldsymbol{\Phi}_{d}\right)$.

Now, in order to proceed with the stability analysis, the following scalar function is defined:

$$
\begin{aligned}
V\left(\tilde{\boldsymbol{\xi}}, \hat{\boldsymbol{\zeta}}, \tilde{\boldsymbol{\theta}}_{d}, \tilde{\boldsymbol{\theta}}_{k}\right)= & \frac{1}{2}\left[\boldsymbol{\varepsilon}^{T} \boldsymbol{X} \boldsymbol{\varepsilon}+\tilde{\boldsymbol{\theta}}_{d}^{T} \boldsymbol{\Gamma}_{d}^{-1} \tilde{\boldsymbol{\theta}}_{d}\right. \\
& \left.+\tilde{\boldsymbol{\theta}}_{k}^{T} \boldsymbol{\Gamma}_{k}^{-1} \tilde{\boldsymbol{\theta}}_{k}\right]+\mathcal{U}_{P}(\tilde{\boldsymbol{\xi}}),
\end{aligned}
$$

where $\varepsilon=\left[\begin{array}{ll}\tilde{\boldsymbol{\xi}}^{T} & \hat{\boldsymbol{\zeta}}^{T}\end{array}\right]^{T}$ and

$$
\boldsymbol{X}=\left[\begin{array}{ll}
\boldsymbol{K}_{D} & \boldsymbol{M}_{d} \\
\boldsymbol{M}_{d} & \boldsymbol{M}_{d}
\end{array}\right]
$$

Observe that by using the Schur complement (Horn and Johnson, 2012)

$$
\begin{aligned}
\boldsymbol{X}>0 \Longleftrightarrow & \boldsymbol{M}_{d}>0 \\
& \text { and } \boldsymbol{K}_{D}-\boldsymbol{M}_{d} \boldsymbol{M}_{d}^{-1} \boldsymbol{M}_{d}>0,
\end{aligned}
$$

and the positivity of $\boldsymbol{X}$ is equivalent to that of $\boldsymbol{M}_{d}$ and $\boldsymbol{K}_{D}-\boldsymbol{M}_{d}$. Since $\boldsymbol{M}_{d}$ and $\boldsymbol{K}_{D}$ are diagonal, positive definite matrices such that inequality (20) is satisfied, we have $\boldsymbol{X}>0$. Under the conditions on the function $\mathcal{U}_{P}(\tilde{\boldsymbol{\xi}})$ 
imposed through (21) and (22), and the quadratic form of the remaining terms, $V\left(\tilde{\boldsymbol{\xi}}, \hat{\boldsymbol{\zeta}}, \tilde{\boldsymbol{\theta}}_{d}, \tilde{\boldsymbol{\theta}}_{k}\right)$ is concluded to be positive definite. Additionally, notice that $\varepsilon^{T} \boldsymbol{X} \varepsilon \rightarrow \infty$ as $\|\tilde{\boldsymbol{\xi}}\| \rightarrow \infty, \boldsymbol{\varepsilon}^{T} \boldsymbol{X} \boldsymbol{\varepsilon} \rightarrow \infty$ as $\|\hat{\boldsymbol{\zeta}}\| \rightarrow \infty, \tilde{\boldsymbol{\theta}}_{d}^{T} \boldsymbol{\Gamma}_{d}^{-1} \tilde{\boldsymbol{\theta}}_{d} \rightarrow$ $\infty$ as $\left\|\tilde{\boldsymbol{\theta}}_{d}\right\| \rightarrow \infty$ and $\tilde{\boldsymbol{\theta}}_{k}^{T} \boldsymbol{\Gamma}_{k}^{-1} \tilde{\boldsymbol{\theta}}_{k} \rightarrow \infty$ as $\left\|\tilde{\boldsymbol{\theta}}_{k}\right\| \rightarrow \infty$, which proves that $V\left(\tilde{\boldsymbol{\xi}}, \hat{\boldsymbol{\zeta}}, \tilde{\boldsymbol{\theta}}_{d}, \tilde{\boldsymbol{\theta}}_{k}\right)$ is radially unbounded (Khalil, 1996).

Furthermore, the upper-right derivative of $V\left(\tilde{\boldsymbol{\xi}}, \hat{\boldsymbol{\zeta}}, \tilde{\boldsymbol{\theta}}_{d}, \tilde{\boldsymbol{\theta}}_{k}\right)$ along the system trajectories, $\dot{V}=D^{+} V$ (Rouche et al., 1977; Michel et al., 2008), is given by

$$
\begin{aligned}
\dot{V}(\tilde{\boldsymbol{\xi}}, \hat{\boldsymbol{\zeta}}, & \left.\tilde{\boldsymbol{\theta}}_{d}, \tilde{\boldsymbol{\theta}}_{k}\right) \\
= & \tilde{\boldsymbol{\xi}}^{T} \boldsymbol{K}_{D} \hat{\boldsymbol{\zeta}}+\tilde{\boldsymbol{\xi}}^{T} \boldsymbol{M}_{d} \dot{\boldsymbol{\zeta}}+\hat{\boldsymbol{\zeta}}^{T} \boldsymbol{M}_{d} \hat{\boldsymbol{\zeta}} \\
& +\hat{\boldsymbol{\zeta}}^{T} \boldsymbol{M}_{d} \dot{\dot{\boldsymbol{\zeta}}}^{2} \tilde{\boldsymbol{\theta}}_{d}^{T} \boldsymbol{\Gamma}_{d}^{-1} \dot{\tilde{\boldsymbol{\theta}}}_{d} \\
& +\tilde{\boldsymbol{\theta}}_{k}^{T} \boldsymbol{\Gamma}_{k}^{-1} \dot{\tilde{\boldsymbol{\theta}}}_{k}+\nabla \mathcal{U}_{P}^{T}(\tilde{\boldsymbol{\xi}}) \hat{\boldsymbol{\zeta}} \\
= & \tilde{\boldsymbol{\xi}}^{T} \boldsymbol{K}_{D} \hat{\boldsymbol{\zeta}}+\hat{\boldsymbol{\zeta}}^{T} \boldsymbol{M}_{d} \hat{\boldsymbol{\zeta}} \\
& +[\tilde{\boldsymbol{\xi}}+\hat{\boldsymbol{\zeta}}]^{T} \boldsymbol{M}_{d}\left[\boldsymbol{\Phi}_{d} \tilde{\boldsymbol{\theta}}_{d}+\boldsymbol{\Phi}_{e} \tilde{\boldsymbol{\theta}}_{k}\right] \\
& -\left[\tilde{\boldsymbol{\xi}}+\hat{\boldsymbol{\zeta}}^{T}\left[\nabla \mathcal{U}_{P}\left(\tilde{\boldsymbol{\xi}}^{T}+\boldsymbol{K}_{D} \hat{\boldsymbol{\zeta}}\right]\right.\right. \\
& -\left[\boldsymbol{\Phi}_{d} \tilde{\boldsymbol{\theta}}_{d}+\boldsymbol{\Phi}_{e} \tilde{\boldsymbol{\theta}}_{k}\right]^{T} \boldsymbol{M}_{d}[\tilde{\boldsymbol{\xi}}+\hat{\boldsymbol{\zeta}}] \\
& -\tilde{\boldsymbol{\theta}}_{k}^{T} \boldsymbol{W}_{k}^{T} \boldsymbol{\Lambda}_{k} \boldsymbol{W}_{k} \tilde{\boldsymbol{\theta}}_{k}+\nabla \mathcal{U}_{P}^{T}(\tilde{\boldsymbol{\xi}}) \hat{\boldsymbol{\zeta}} \\
= & -\tilde{\boldsymbol{\xi}}^{T} \nabla \mathcal{U}_{P}(\tilde{\boldsymbol{\xi}})-\hat{\boldsymbol{\zeta}}^{T}\left[\boldsymbol{K}_{D}-\boldsymbol{M}_{d}\right] \hat{\boldsymbol{\zeta}} \\
& -\tilde{\boldsymbol{\theta}}_{k}^{T} \boldsymbol{W}_{k}^{T} \boldsymbol{\Lambda}_{k} \boldsymbol{W}_{k} \tilde{\boldsymbol{\theta}}_{k},
\end{aligned}
$$

where $\hat{\dot{\boldsymbol{\zeta}}}, \quad \dot{\tilde{\boldsymbol{\theta}}}_{d}$ and $\dot{\tilde{\boldsymbol{\theta}}}_{k}$ have been replaced by the expressions 28a-28c from the closed-loop dynamics. Therefore, under Assumption 11, the inequality (20) and the conditions on the function $\mathcal{U}_{P}(\tilde{\boldsymbol{\xi}})$ stated through the expressions (23)-(24), $\dot{V}\left(\tilde{\boldsymbol{\xi}}, \hat{\boldsymbol{\zeta}}, \tilde{\boldsymbol{\theta}}_{d}, \tilde{\boldsymbol{\theta}}_{k}\right) \leq$ $0 \forall\left(\tilde{\boldsymbol{\xi}}, \hat{\boldsymbol{\zeta}}, \tilde{\boldsymbol{\theta}}_{d}, \tilde{\boldsymbol{\theta}}_{k}\right) \in \mathbb{R}^{m} \times \mathbb{R}^{m} \times \mathbb{R}^{p} \times \mathbb{R}^{r}$, with $\dot{V}\left(\tilde{\boldsymbol{\xi}}, \hat{\boldsymbol{\zeta}}, \tilde{\boldsymbol{\theta}}_{d}, \tilde{\boldsymbol{\theta}}_{k}\right)=0 \Leftrightarrow\left(\tilde{\boldsymbol{\xi}}, \hat{\boldsymbol{\zeta}}, \tilde{\boldsymbol{\theta}}_{k}\right)=\left(\mathbf{0}_{m}, \mathbf{0}_{m}, \mathbf{0}_{r}\right)$.

Now, the second upper-right derivative of $V\left(\tilde{\boldsymbol{\xi}}, \hat{\boldsymbol{\zeta}}, \tilde{\boldsymbol{\theta}}_{d}, \tilde{\boldsymbol{\theta}}_{k}\right)$ along the system trajectories, i.e., $\ddot{V}=D^{+} \dot{V}$, is given by

$$
\begin{aligned}
& \ddot{V}\left(\tilde{\boldsymbol{\xi}}, \hat{\boldsymbol{\zeta}}, \tilde{\boldsymbol{\theta}}_{d}, \tilde{\boldsymbol{\theta}}_{k}\right) \\
&=-\hat{\boldsymbol{\zeta}}^{T} \nabla \mathcal{U}_{P}(\tilde{\boldsymbol{\xi}})-\tilde{\boldsymbol{\xi}}^{T} \boldsymbol{H}_{P}(\tilde{\boldsymbol{\xi}}) \hat{\boldsymbol{\zeta}} \\
&-2 \hat{\boldsymbol{\zeta}}^{T}\left[\boldsymbol{K}_{D}-\boldsymbol{M}_{d}\right] \hat{\dot{\boldsymbol{\zeta}}} \\
&-2 \tilde{\boldsymbol{\theta}}_{k}^{T} \boldsymbol{W}_{k}^{T} \boldsymbol{\Lambda}_{k}\left[\dot{\boldsymbol{W}}_{k} \tilde{\boldsymbol{\theta}}_{k}+\boldsymbol{W}_{k} \dot{\tilde{\boldsymbol{\theta}}}_{k}\right] \\
&=-\hat{\boldsymbol{\zeta}}^{T} \nabla \mathcal{U}_{P}(\tilde{\boldsymbol{\xi}})-\tilde{\boldsymbol{\xi}}^{T} \boldsymbol{H}_{P}(\tilde{\boldsymbol{\xi}}) \hat{\boldsymbol{\zeta}} \\
&-2 \hat{\boldsymbol{\zeta}}^{T}\left[\boldsymbol{K}_{D}-\boldsymbol{M}_{d}\right]\left[\boldsymbol{\Phi}_{d} \tilde{\boldsymbol{\theta}}_{d}+\boldsymbol{\Phi}_{e} \tilde{\boldsymbol{\theta}}_{k}\right. \\
&\left.-\boldsymbol{M}_{d}^{-1}\left[\nabla \mathcal{U}_{P}(\tilde{\boldsymbol{\xi}})+\boldsymbol{K}_{D} \hat{\boldsymbol{\zeta}}\right]\right]
\end{aligned}
$$

$$
\begin{aligned}
& -2 \tilde{\boldsymbol{\theta}}_{k}^{T} \boldsymbol{W}_{k}^{T} \boldsymbol{\Lambda}_{k}\left[\dot{\boldsymbol{W}}_{k} \tilde{\boldsymbol{\theta}}_{k}\right. \\
& -\boldsymbol{W}_{k} \boldsymbol{\Gamma}_{k}\left[\boldsymbol{\Phi}_{e}^{T} \boldsymbol{M}_{d}[\tilde{\boldsymbol{\xi}}+\hat{\boldsymbol{\zeta}}]\right. \\
& \left.\left.+\boldsymbol{W}_{k}^{T} \boldsymbol{\Lambda}_{k} \boldsymbol{W}_{k} \tilde{\boldsymbol{\theta}}_{k}\right]\right]
\end{aligned}
$$

where

$$
\boldsymbol{H}_{P}(\tilde{\boldsymbol{\xi}})=\left[\begin{array}{cccc}
\frac{\partial^{2} \mathcal{U}_{P}}{\partial \tilde{\xi}_{1}^{2}} & \frac{\partial^{2} \mathcal{U}_{P}}{\partial \tilde{\xi}_{1} \partial \tilde{\xi}_{2}} & \cdots & \frac{\partial^{2} \mathcal{U}_{P}}{\partial \tilde{\xi}_{1} \partial \tilde{\xi}_{m}} \\
\frac{\partial^{2} \mathcal{U}_{P}}{\partial \tilde{\xi}_{2} \partial \tilde{\xi}_{1}} & \frac{\partial^{2} \mathcal{U}_{P}}{\partial \tilde{\xi}_{2}^{2}} & \cdots & \frac{\partial^{2} \mathcal{U}_{P}}{\partial \tilde{\xi}_{2} \partial \tilde{\xi}_{m}} \\
\vdots & \vdots & \ddots & \vdots \\
\frac{\partial^{2} \mathcal{U}_{P}}{\partial \tilde{\xi}_{m} \partial \tilde{\xi}_{1}} & \frac{\partial^{2} \mathcal{U}_{P}}{\partial \tilde{\xi}_{m} \partial \tilde{\xi}_{2}} & \cdots & \frac{\partial^{2} \mathcal{U}_{P}}{\partial \tilde{\xi}_{m}^{2}}
\end{array}\right]
$$

represents the Hessian matrix of $\mathcal{U}_{P}(\tilde{\boldsymbol{\xi}})$. Assume that $\boldsymbol{x}_{d}, \dot{\boldsymbol{x}}_{d}, \ddot{\boldsymbol{x}}_{d}, \boldsymbol{f}_{e} \in \mathcal{L}_{\infty}^{m}$. Then, from Eqn. (32) $\dot{V}\left(\tilde{\boldsymbol{\xi}}, \hat{\boldsymbol{\zeta}}, \tilde{\boldsymbol{\theta}}_{d}, \tilde{\boldsymbol{\theta}}_{k}\right) \leq 0 \Rightarrow \tilde{\boldsymbol{\xi}}, \hat{\boldsymbol{\zeta}} \in \mathcal{L}_{2}^{m} \cap \mathcal{L}_{\infty}^{m}, \boldsymbol{W}_{k} \tilde{\boldsymbol{\theta}}_{k} \in \mathcal{L}_{2}^{r}$ and $\tilde{\boldsymbol{\theta}}_{d} \in \mathcal{L}_{\infty}^{p}, \tilde{\boldsymbol{\theta}}_{k} \in \mathcal{L}_{\infty}^{r}$. Further, from Eqns. 110, (27) and the features of $\mathcal{U}_{P}$ defined in 21)-24), we have that $\tilde{\boldsymbol{\xi}}, \hat{\boldsymbol{\zeta}} \in \mathcal{L}_{\infty}^{m} \Rightarrow \tilde{\boldsymbol{x}}, \boldsymbol{x}_{f e}, \dot{\boldsymbol{x}}_{f e}, \nabla \mathcal{U}_{P} \in \mathcal{L}_{\infty}^{m}, \boldsymbol{H}_{P} \in \mathcal{L}_{\infty}^{m \times m}$ and $\boldsymbol{x}, \hat{\dot{\boldsymbol{x}}} \in \mathcal{L}_{\infty}^{m}$. Also, as $\boldsymbol{W}_{k}$ is bounded, $\tilde{\boldsymbol{\theta}}_{k} \in \mathcal{L}_{\infty}^{r}$ implies $\boldsymbol{\theta}_{k} \in \mathcal{L}_{\infty}^{r}$ so that $\boldsymbol{z}, \dot{\boldsymbol{x}} \in \mathcal{L}_{\infty}^{m}$ in Eqns. (2) and (5). Thus, $\dot{\boldsymbol{q}} \in \mathcal{L}_{\infty}^{n}$ and under Assumption 1, $\boldsymbol{J}(\boldsymbol{q})$ is a bounded nonsingular matrix, which implies that $\boldsymbol{Y}_{e} \in$ $\mathcal{L}_{\infty}^{n \times r}$ in Eqn. (9). Then $\boldsymbol{\Phi}_{e}$ is a bounded matrix, since $\hat{\boldsymbol{M}}_{0}^{-1}$ is bounded according to Property 2 and $\hat{\dot{\boldsymbol{x}}} \in \mathcal{L}_{\infty}^{m}$ implies $\hat{\boldsymbol{J}} \in \mathcal{L}_{\infty}^{m \times n}$. On the other hand, from the previous results and Properties 2 and $3, \boldsymbol{\Phi}_{d}$ is a bounded matrix. Therefore, with bounded gain matrices $\boldsymbol{K}_{D}, \boldsymbol{M}_{d}, \boldsymbol{\Lambda}_{k}$ and $\boldsymbol{\Gamma}_{k}$, we have that

$$
\begin{aligned}
\dot{\boldsymbol{x}}_{d}-\dot{\boldsymbol{x}}-\dot{\boldsymbol{x}}_{f e} & =\dot{\tilde{\boldsymbol{\xi}}} \in \mathcal{L}_{\infty}^{m}, \\
\ddot{V}\left(\tilde{\boldsymbol{\xi}}, \hat{\boldsymbol{\zeta}}, \tilde{\boldsymbol{\theta}}_{d}, \tilde{\boldsymbol{\theta}}_{k}\right) & \in \mathcal{L}_{\infty},
\end{aligned}
$$

and according to the Barbalat lemma (Slotine et al., 1991), $\tilde{\boldsymbol{\xi}} \rightarrow \mathbf{0}_{m}$ and $\dot{\tilde{\xi}} \rightarrow \mathbf{0}_{m}$ as $t \rightarrow \infty$, which completes the proof.

Remark 1. Let $\boldsymbol{K}_{P} \in \mathbb{R}^{m \times m}$ be a positive definite diagonal matrix, i.e., $\boldsymbol{K}_{P}=\operatorname{diag}\left[k_{P 1}, \ldots, k_{P m}\right]$ with $k_{P i}>0$ for all $i=1,2, \ldots, m$. An adaptive PD-type impedance controller is obtained from (19) by selecting

$$
\mathcal{U}_{P}(\tilde{\boldsymbol{\xi}})=\frac{1}{2} \tilde{\boldsymbol{\xi}}^{T} \boldsymbol{K}_{P} \tilde{\boldsymbol{\xi}}
$$

such that

$$
\nabla \mathcal{U}_{p}(\tilde{\boldsymbol{\xi}})=\boldsymbol{K}_{P} \tilde{\boldsymbol{\xi}}
$$

Additionally, an adaptive Tanh(P)D-type controller can be retrieved from (19) by defining

$$
\mathcal{U}_{P}(\tilde{\boldsymbol{\xi}})=\boldsymbol{f}_{P}(\tilde{\boldsymbol{\xi}})^{T} \boldsymbol{K}_{P} \boldsymbol{f}_{P}(\tilde{\boldsymbol{\xi}}),
$$

where

$$
\boldsymbol{f}_{P}(\tilde{\boldsymbol{\xi}})=\left[\begin{array}{c}
\sqrt{\ln \left\{\cosh \left(\tilde{\xi}_{1}\right)\right\}} \\
\vdots \\
\sqrt{\ln \left\{\cosh \left(\tilde{\xi}_{m}\right)\right\}}
\end{array}\right]
$$


Therefore, $\mathcal{U}_{P}$ has a quadratic form to ensure positivity of the function. The gradient of $\mathcal{U}_{P}$ is given by

$$
\nabla \mathcal{U}_{P}(\tilde{\boldsymbol{\xi}})=2 \frac{\partial \boldsymbol{f}_{P}(\tilde{\boldsymbol{\xi}})}{\partial \tilde{\boldsymbol{\xi}}} \boldsymbol{K}_{P} \boldsymbol{f}_{P}(\tilde{\boldsymbol{\xi}}),
$$

where

$$
\frac{\partial \boldsymbol{f}_{P}(\tilde{\boldsymbol{\xi}})}{\partial \tilde{\boldsymbol{\xi}}}=\frac{1}{2}\left[\begin{array}{ccc}
\frac{\tanh \left(\tilde{\xi}_{1}\right)}{\sqrt{\ln \left\{\cosh \left(\tilde{\xi}_{1}\right)\right\}}} & & 0 \\
& \ddots & \\
0 & & \frac{\tanh \left(\tilde{\xi}_{m}\right)}{\sqrt{\ln \left\{\cosh \left(\tilde{\xi}_{m}\right)\right\}}}
\end{array}\right] .
$$

Then

$$
\nabla \mathcal{U}_{P}(\tilde{\boldsymbol{\xi}})=\boldsymbol{K}_{P} \tanh (\tilde{\boldsymbol{\xi}})
$$

with $\tanh (\tilde{\boldsymbol{\xi}})=\left[\tanh \left(\tilde{\xi}_{1}\right), \ldots, \tanh \left(\tilde{\xi}_{m}\right)\right]^{T}$.

\section{Simulation results}

The validation of the proposed adaptive impedance control scheme has been oriented towards verifying its efficiency for constrained and unconstrained path tracking. The simulation control tests have been implemented using the model of a 2-DOF direct-drive robot manipulator. The system consists of a 2-revolute-joint robot arm characterized by Reyes and Kelly (1997). It is composed of two links actuated by direct-drive brushless servomotors whose torque limits are $T_{1}=150 \mathrm{Nm}$ and $T_{2}=15 \mathrm{Nm}$, respectively. The corresponding kinematic and dynamic parameters are presented in Table 1

Table 1. Kinematic and dynamic parameters of the robot manipulator.

\begin{tabular}{|c|c|c|}
\hline Parameter & Value & Units \\
\hline \hline$\theta_{k 1}$ & 0.450 & $\mathrm{~m}$ \\
$\theta_{k 2}$ & 0.450 & $\mathrm{~m}$ \\
$\theta_{d 1}$ & 2.351 & $\mathrm{Nm} \mathrm{s}^{2} / \mathrm{rad}$ \\
$\theta_{d 2}$ & 0.084 & $\mathrm{Nm} \mathrm{s}^{2} / \mathrm{rad}$ \\
$\theta_{d 3}$ & 0.102 & $\mathrm{Nm} \mathrm{s}^{2} / \mathrm{rad}$ \\
$\theta_{d 4}$ & 38.465 & $\mathrm{Nm}$ \\
$\theta_{d 5}$ & 1.825 & $\mathrm{Nm}$ \\
\hline
\end{tabular}

The robot structure and the kinematic data imply the following Jacobian matrix:

$$
\boldsymbol{J}(\boldsymbol{q})=\left[\begin{array}{cc}
\theta_{k 1} \mathrm{c}\left(q_{1}\right)+\theta_{k 2} \mathrm{c}\left(q_{1}+q_{2}\right) & \theta_{k 2} \mathrm{c}\left(q_{1}+q_{2}\right) \\
\theta_{k 1} \mathrm{~s}\left(q_{1}\right)+\theta_{k 2} \mathrm{~s}\left(q_{1}+q_{2}\right) & \theta_{k 2} \mathrm{~s}\left(q_{1}+q_{2}\right)
\end{array}\right],
$$

where $\theta_{k 1}$ and $\theta_{k 2}$ are the lengths of the robot links (i.e., $r=2), \mathrm{c}(\cdot)$ and $\mathrm{s}(\cdot)$ denote the $\cos (\cdot)$ and $\sin (\cdot)$ functions, respectively, and $\boldsymbol{q}=\left[\begin{array}{ll}q_{1} & q_{2}\end{array}\right]^{T}$ represents the vector of joint positions. According to Property 1, the kinematic regression matrix is given by

$$
\boldsymbol{Y}_{k}(\boldsymbol{q}, \dot{\boldsymbol{q}})=\left[\begin{array}{ll}
\dot{q}_{1} \cos \left(q_{1}\right) & {\left[\dot{q}_{1}+\dot{q}_{2}\right] \cos \left(q_{1}+q_{2}\right)} \\
\dot{q}_{1} \sin \left(q_{1}\right) & {\left[\dot{q}_{1}+\dot{q}_{2}\right] \sin \left(q_{1}+q_{2}\right)}
\end{array}\right] .
$$

On the other hand, the vector of dynamic parameters is $\boldsymbol{\theta}_{d}=\left[\begin{array}{lllll}\theta_{d 1} & \theta_{d 2} & \theta_{d 3} & \theta_{d 4} & \theta_{d 5}\end{array}\right]^{T}$ ( i.e., $p=5$ ), where $\theta_{d 1}=m_{1} l_{c 1}^{2}+m_{2}\left(l_{1}^{2}+l_{c 2}^{2}\right)+I_{1}+I_{2}, \theta_{d 2}=m_{2} l_{1} l_{c 2}$, $\theta_{d 3}=m_{2} l_{c 2}^{2}+I_{2}, \theta_{d 4}=g\left[m_{1} l_{c 1}+m_{2} l_{1}\right]$ and $\theta_{d 5}=$ $g m_{2} l_{c 2}$, with $m_{i}$ representing the mass, $l_{i}$ the length, $l_{c i}$ the center of mass, and $I_{i}$ the moment of inertia for the $i$-th link. Here $g=9.81 \mathrm{~m} / \mathrm{s}^{2}$ represents the gravitational acceleration. The dynamic model of the robot manipulator is composed of

$$
\begin{aligned}
\boldsymbol{M}(\boldsymbol{q}) & =\left[\begin{array}{cc}
\theta_{d 1}+2 \theta_{d 2} \cos \left(q_{2}\right) & \theta_{d 3}+\theta_{d 2} \cos \left(q_{2}\right) \\
\theta_{d 3}+\theta_{d 2} \cos \left(q_{2}\right) & \theta_{d 3}
\end{array}\right], \\
\boldsymbol{C}(\boldsymbol{q}, \dot{\boldsymbol{q}}) & =\left[\begin{array}{cc}
-2 \theta_{d 2} \sin \left(q_{2}\right) \dot{q}_{2} & -\theta_{d 2} \sin \left(q_{2}\right) \dot{q}_{2} \\
\theta_{d 2} \sin \left(q_{2}\right) \dot{q}_{1} & 0
\end{array}\right], \\
\boldsymbol{g}(\boldsymbol{q}) & =\left[\begin{array}{c}
\theta_{d 4} \sin \left(q_{1}\right)+\theta_{d 5} \sin \left(q_{1}+q_{2}\right) \\
\theta_{d 5} \sin \left(q_{1}+q_{2}\right)
\end{array}\right]
\end{aligned}
$$

and, according to Property 3

$$
\begin{aligned}
& \boldsymbol{Y}_{d}(\boldsymbol{q}, \dot{\boldsymbol{q}}, \ddot{\boldsymbol{q}}) \\
& \quad=\left[\begin{array}{lllcl}
\ddot{q}_{1} & y_{d 12} & \ddot{q}_{2} & \mathrm{~s}\left(q_{1}\right) & \mathrm{s}\left(q_{1}+q_{2}\right) \\
0 & y_{d 22} & \ddot{q}_{1}+\ddot{q}_{2} & 0 & \mathrm{~s}\left(q_{1}+q_{2}\right)
\end{array}\right],
\end{aligned}
$$

where

$$
\begin{aligned}
& y_{d 12}=\left[2 \ddot{q}_{1}+\ddot{q}_{2}\right] \cos \left(q_{2}\right)-\left[2 \dot{q}_{1}+\dot{q}_{2}\right] \dot{q}_{2} \sin \left(q_{2}\right), \\
& y_{d 22}=\ddot{q}_{1} \cos \left(q_{2}\right)+\dot{q}_{1}^{2} \sin \left(q_{2}\right) .
\end{aligned}
$$

In turn, the regression matrix for the interaction torques is given by

$$
\boldsymbol{Y}_{e}\left(\boldsymbol{q}, \boldsymbol{f}_{e}\right)=\left[\begin{array}{cc}
y_{e 11} & y_{e 12} \\
0 & y_{e 22}
\end{array}\right]
$$

with

$$
\begin{aligned}
& y_{e 11}=f_{e 1} \cos \left(q_{1}\right)+f_{e 2} \sin \left(q_{1}\right), \\
& y_{e 12}=f_{e 1} \cos \left(q_{1}+q_{2}\right)+f_{e 2} \sin \left(q_{1}+q_{2}\right), \\
& y_{e 22}=f_{e 1} \cos \left(q_{1}+q_{2}\right)+f_{e 2} \sin \left(q_{1}+q_{2}\right),
\end{aligned}
$$

In order to include non-modeled dynamics, which allows us to validate the adaptability of the control scheme, the following frictional forces were considered:

$$
\begin{aligned}
& f_{r 1}=2.288 \dot{q}_{1}+f_{c 1} \operatorname{sign}\left(\dot{q}_{1}\right), \\
& f_{r 2}=0.175 \dot{q}_{2}+1.734 \operatorname{sign}\left(\dot{q}_{2}\right),
\end{aligned}
$$

where $f_{c 1}=7.17$ if $\dot{q}_{1}>0$ and $f_{c 1}=8.049$ if $\dot{q}_{1}<0$ (Reyes and Kelly, 1997).

The proposed adaptive impedance control scheme in Eqns. 16- 19) and 25 was tested in its PD-type form for unconstrained and constrained path tracking. 
5.1. Test 1: Unconstrained path tracking. The following Lissajous curve was selected as the desired trajectory for the unconstrained test

$$
\boldsymbol{x}_{d}(t)=\left[\begin{array}{l}
x_{o 1}+\rho \sin \left(\omega_{a} t\right) \\
x_{o 2}+\rho \sin \left(\omega_{b} t\right)
\end{array}\right],
$$

where $x_{o}=\left[\begin{array}{ll}0.35 & -0.4\end{array}\right]^{T} \mathrm{~m}, \rho=0.25 \mathrm{~m}, \omega_{a}=3$ $\mathrm{rad} / \mathrm{s}$ and $\omega_{b}=2 \mathrm{rad} / \mathrm{s}$. The initial location of the robot end-effector was chosen as $\boldsymbol{x}(0)=[0.45-0.45]^{T} \mathrm{~m}$. The initial values of the dynamic and kinematic parameters were set as $\hat{\boldsymbol{\theta}}_{d}(0)=\left[\begin{array}{lllll}2.0 & 0.15 & 0.15 & 30.0 & 1.0\end{array}\right]^{T}$ and $\hat{\boldsymbol{\theta}}_{k}(0)=\left[\begin{array}{ll}0.5 & 0.6\end{array}\right]^{T}$, respectively. In practice, one of the limitations of the proposed control scheme is that joint acceleration measurements are required. Then, in order to avoid the need for such measurements, accelerations were estimated by filtering the velocity signals (Wang and Xie, 2009), such that $\boldsymbol{\alpha} \triangleq \hat{\ddot{\boldsymbol{q}}}=\left[s /\left(\lambda_{a} s+1\right)\right] \dot{\boldsymbol{q}}$ with $\lambda_{a}=1 / 60$. Therefore, the vector $\boldsymbol{\alpha}$ replaces $\ddot{\boldsymbol{q}}$ in the control structure defined in Eqns. (16)-19) and (25). Then the closed-loop dynamics is now given by

$$
\begin{aligned}
\hat{\dot{\boldsymbol{\zeta}}}= & \hat{\boldsymbol{\Phi}}_{d} \tilde{\boldsymbol{\theta}}_{d}+\boldsymbol{\Phi}_{e} \tilde{\boldsymbol{\theta}}_{k}-\boldsymbol{M}_{d}^{-1}\left[\nabla \mathcal{U}_{P}+\boldsymbol{K}_{D} \hat{\boldsymbol{\zeta}}\right] \\
& +\lambda_{a} \boldsymbol{\Phi}_{M} \dot{\boldsymbol{\alpha}}, \\
\dot{\tilde{\boldsymbol{\theta}}}_{d}= & -\boldsymbol{\Gamma}_{d} \hat{\boldsymbol{\Phi}}_{d}^{T} \boldsymbol{M}_{d}[\tilde{\boldsymbol{\xi}}+\hat{\boldsymbol{\zeta}}], \\
\dot{\tilde{\boldsymbol{\theta}}}_{k}= & -\boldsymbol{\Gamma}_{k}\left[\boldsymbol{\Phi}_{e}^{T} \boldsymbol{M}_{d}[\tilde{\boldsymbol{\xi}}+\hat{\boldsymbol{\zeta}}]+\boldsymbol{W}_{k}^{T} \boldsymbol{\Lambda}_{k} \boldsymbol{W}_{k} \tilde{\boldsymbol{\theta}}_{k}\right],
\end{aligned}
$$

where $\hat{\boldsymbol{\Phi}}_{d} \triangleq \hat{\boldsymbol{J}}(\boldsymbol{q}) \hat{\boldsymbol{M}}_{0}^{-1}(\boldsymbol{q}) \boldsymbol{Y}_{d}(\boldsymbol{q}, \dot{\boldsymbol{q}}, \boldsymbol{\alpha}), \quad \boldsymbol{\Phi}_{M} \triangleq$ $\hat{\boldsymbol{J}}(\boldsymbol{q}) \hat{\boldsymbol{M}}_{0}^{-1}(\boldsymbol{q}) \boldsymbol{M}(\boldsymbol{q})$. Note that $\lambda_{a} \boldsymbol{\Phi}_{M} \dot{\boldsymbol{\alpha}}$ is the additional term in the closed-loop dynamics of the system; because it is possible to verify that this term is bounded and close to zero, a proper tuning of the controller parameters can counteract this additional dynamic uncertainty by preserving (in practice) the stability properties of the proposed scheme.

The parameters of the proposed controller were selected as $\boldsymbol{K}_{P}=15000 \boldsymbol{I}_{m}, \boldsymbol{K}_{D}=2000 \boldsymbol{I}_{m}, \boldsymbol{M}_{d}=$ $2 \boldsymbol{I}_{m}, \boldsymbol{\Gamma}_{d}=\operatorname{diag}\{2000,0.007,0.8,200,1.5\}, \boldsymbol{\Gamma}_{k}=$ $1.2 \boldsymbol{I}_{r}, \boldsymbol{\Lambda}_{k}=50 \boldsymbol{I}_{r}, \lambda=10$. Note that $\boldsymbol{K}_{d}$ and $\boldsymbol{B}_{d}$ are not required for unconstrained path-tracking tasks.

To illustrate the performance of the adaptive controller, the time evolution of the impedance error was recorded. The components of the impedance error are shown in Fig. 11. It is to be noted that the error components tend to a neighborhood of zero as a result of the estimation of accelerations; however, by retuning the control gains the steady-state error can be reduced and so the impedance-control objective is properly fulfilled by ensuring the convergence of the impedance error towards zero as time evolves. It is important to note that in this test there is no interaction or contact with the environment, and hence the impedance error is equal to the tracking error in the Cartesian space.
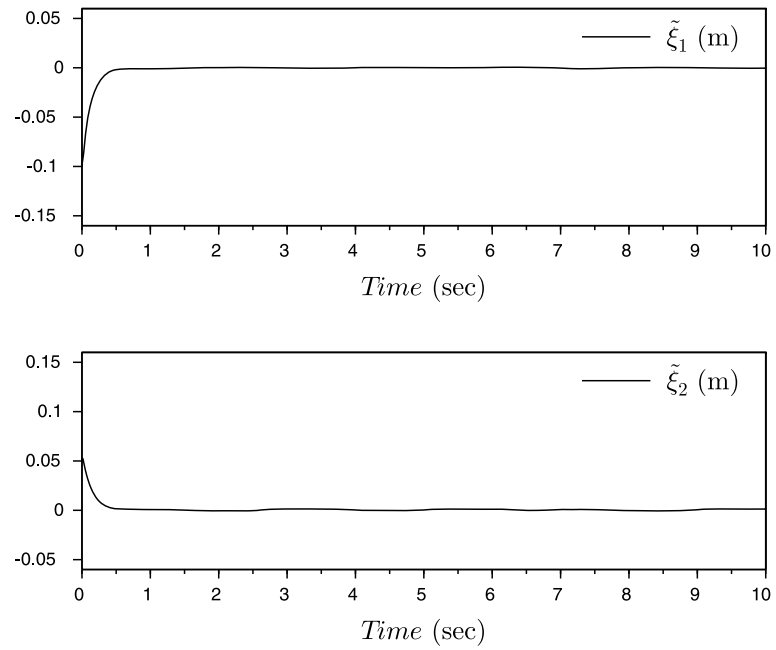

Fig. 1. Test 1: components of the impedance error.

Figure 2 shows the desired and actual end-effector paths. It is possible to observe that the desired trajectory is accurately followed by the robot end-effector, after a short transient period.

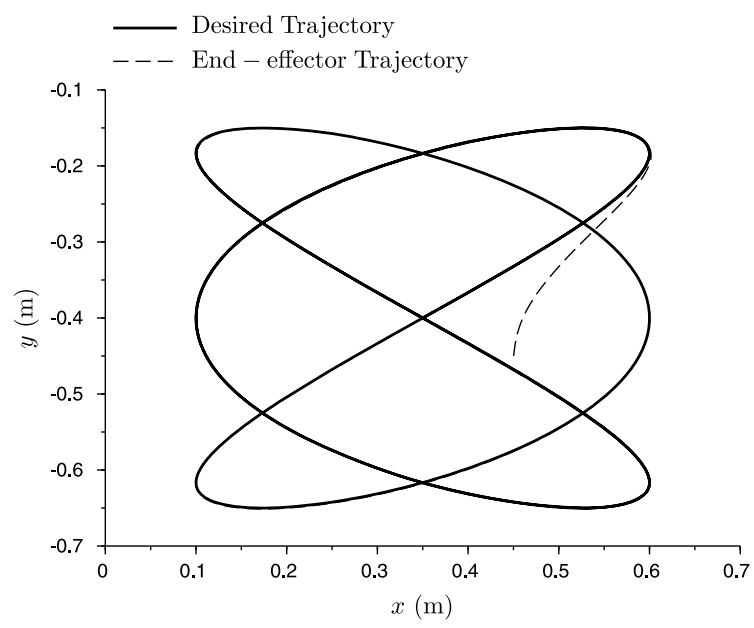

Fig. 2. Test 1: trajectory of the robot end-effector on $x-y$ plane.

Figures 3 and 4 show that, as time evolves, the estimates of kinematic and dynamic parameters remain bounded as expected, based on the stability analysis of the proposed controller. Therefore, it can be concluded that the control scheme works properly when tracking unconstrained trajectories. The initial values $\hat{\boldsymbol{\theta}}_{d}(0)$ and $\hat{\boldsymbol{\theta}}_{k}(0)$ were selected close to the values identified by Reyes and Kelly (1997), therefore, the procedure to be followed would be to perform a process of prior identification of the robotic system parameters and then use the results as initial values. According to the stability analysis presented, it is expected that the kinematic parameters $\hat{\boldsymbol{\theta}}_{k}$ converge to values close to the real ones, regardless 
of the initial conditions $\hat{\boldsymbol{\theta}}_{k}(0)$. However, the dynamic parameters $\hat{\boldsymbol{\theta}}_{d}$ will only have a bounded behavior and they will converge to different values that depend on the initial conditions. This expected behavior is validated by the results obtained and presented in the Figs. 3 and 4
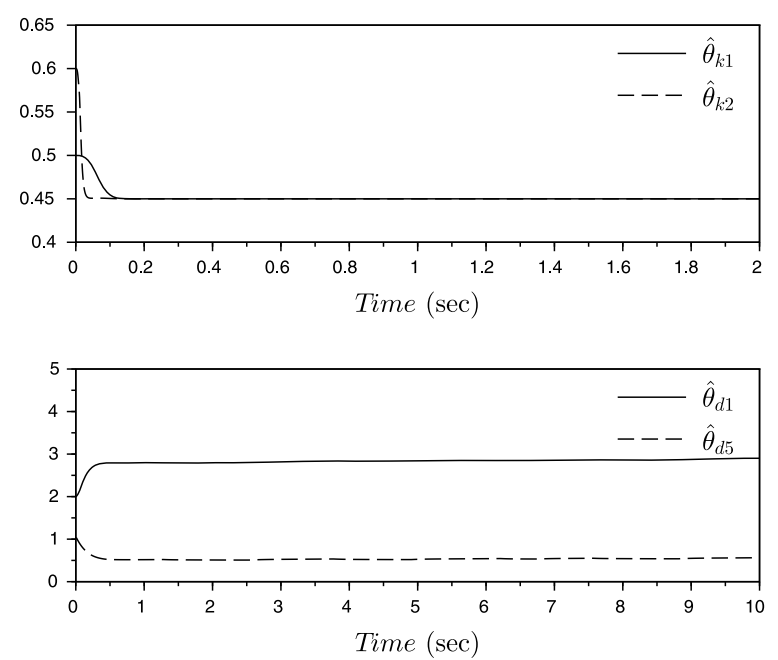

Fig. 3. Test 1: time evolution of the estimates of kinematic and dynamic parameters, respectively.
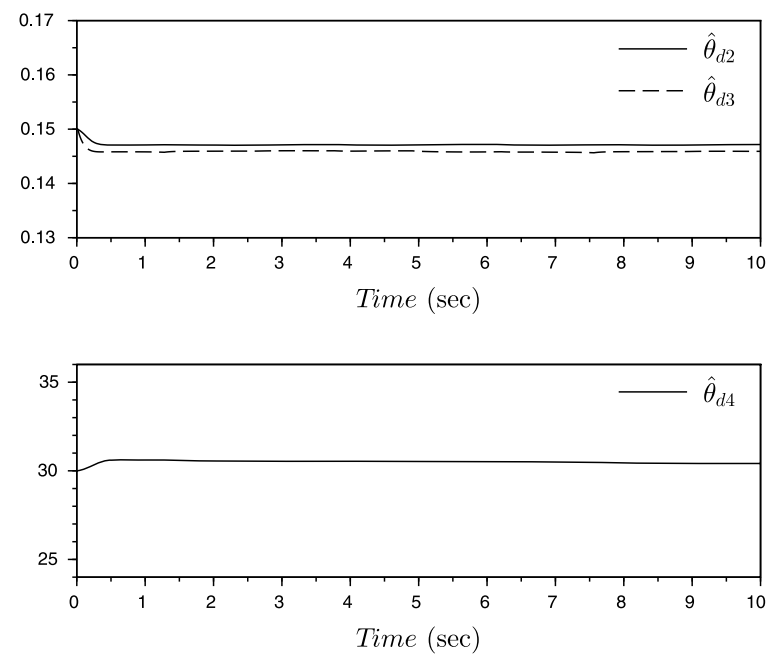

Fig. 4. Test 1: time evolution of the estimates of dynamic parameters.

5.2. Test 2: Constrained path tracking. In order to test the performance of the impedance controller regarding the tracking of constrained paths during the robot-environment interaction, a rigid ring corresponding to the following path was considered:

$$
\boldsymbol{x}_{d}(t)=\left[\begin{array}{l}
x_{o 1}+\rho \cos (\omega t) \\
x_{o 2}-\rho \sin (\omega t)
\end{array}\right],
$$

where $\boldsymbol{x}_{o}=\left[\begin{array}{ll}0.5 & -0.4\end{array}\right]^{T} \mathrm{~m}, \rho=0.25 \mathrm{~m}$ and $\omega=\pi / 4$ $\mathrm{rad} / \mathrm{s}$. The interaction with the ring was modeled as

$$
f_{e}=k_{e}\left[\left\|\boldsymbol{x}-\boldsymbol{x}_{o}\right\|-\rho_{e}\right],
$$

where $k_{e}=5000 \mathrm{~N} / \mathrm{m}$ is the stiffness of the surface and $\rho_{e}=0.24 \mathrm{~m}$ is the location of the ring surface measured from $\boldsymbol{x}_{o}$. Then the components of the contact force $\boldsymbol{f}_{e}$ were computed as

$$
\boldsymbol{f}_{e}=\left[\begin{array}{l}
f_{e} \cos (\phi) \\
f_{e} \sin (\phi)
\end{array}\right]
$$

with

$$
\phi=\tan ^{-1}\left(\frac{x_{2}-x_{o 2}}{x_{1}-x_{o 1}}\right) .
$$

The initial values of the dynamic and kinematic parameters and the controller gains $\boldsymbol{K}_{P}, \boldsymbol{K}_{D}, \boldsymbol{M}_{d}, \boldsymbol{\Lambda}_{k}$ and $\lambda$ were the same used in the unconstrained-motion test. The initial location of the robot end-effector was $\boldsymbol{x}(0)=\left[\begin{array}{ll}0.5 & -0.64\end{array}\right]^{T} \mathrm{~m}$, and the rest of controller parameters were selected as $\boldsymbol{B}_{d}=50 \boldsymbol{I}_{m}, \boldsymbol{K}_{d}=150 \boldsymbol{I}_{m}$, $\boldsymbol{\Gamma}_{k}=5 \boldsymbol{I}_{r}$ and $\boldsymbol{\Gamma}_{d}=\operatorname{diag}\{30000,0.1,5,500,2.5\}$.

First, the errors obtained for the tracking of the desired trajectory and the interaction with the surface (impedance error) are presented. In Fig. 5, it can be observed that the tracking error $\tilde{\boldsymbol{x}}$ is different from zero because the interaction of the robot end effector with the surface prevents it from reaching the desired trajectory. However, the dynamic behavior of the system is correctly regulated to successfully interact with the environment. It is to be noted that the impedance-control objective is properly achieved and the convergence of the impedance error $\tilde{\xi}$ to zero as time evolves is ensured.
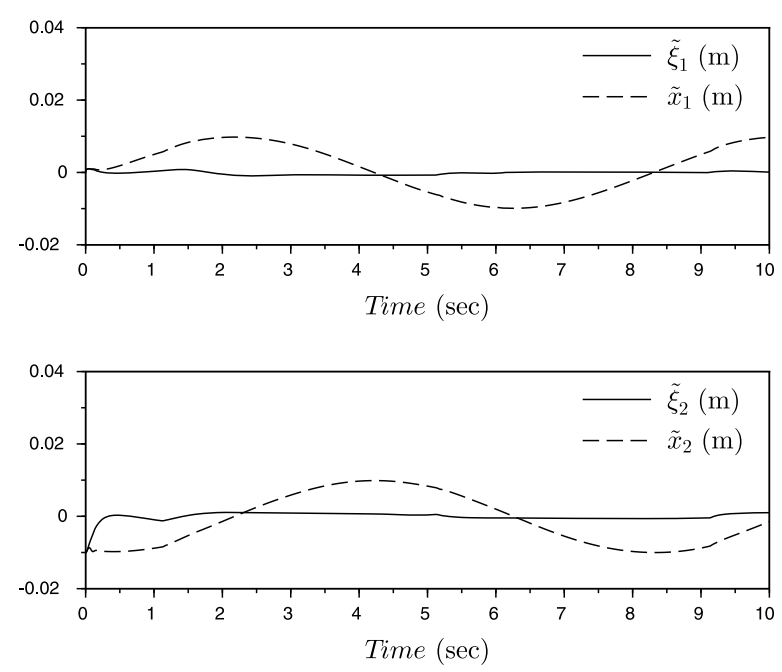

Fig. 5. Test 2: components of the impedance and tracking errors, respectively.

The correct performance of the controller can be verified in Fig. 6, which displays the desired trajectory 


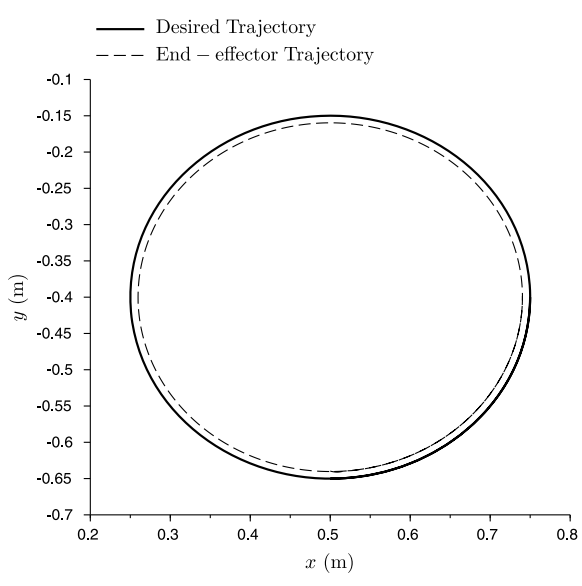

Fig. 6. Test 2: trajectory of the robot end-effector on the $x-y$ plane.
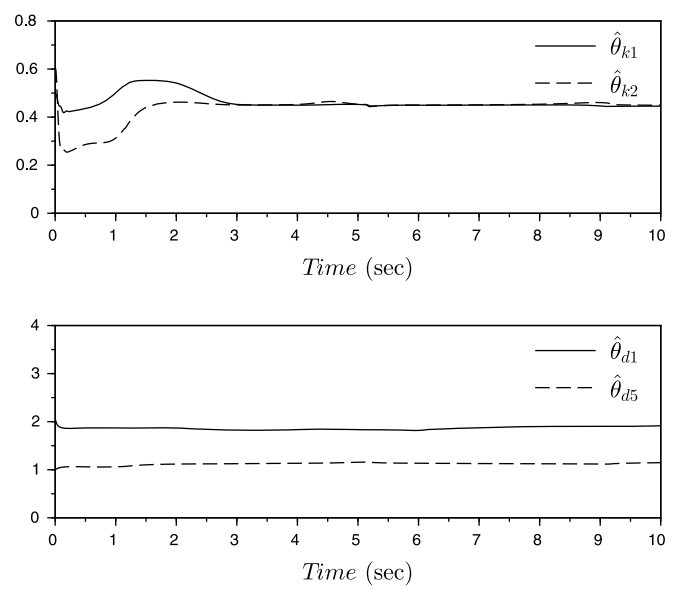

Fig. 7. Test 2: time evolution of the estimates of kinematic and dynamic parameters, respectively.

that was pre-planned in accordance with (41) for a radius of $\rho=0.25 \mathrm{~m}$, and the trajectory followed by the robot, for a radius of $\rho_{e}=0.24 \mathrm{~m}$, corresponding to the path imposed by the surface of the environment.

In the same way as in Test 1, the time evolution of the kinematic and dynamic parameters remains bounded as shown in Fig. 7 and 8 . Finally, in Fig. 9, the components of the interaction force are presented, which are continuous signals and in this way it can be verified that the robot end effector remains in contact with the surface during the whole movement.

\section{Conclusions}

In this paper, an adaptive impedance control scheme for robot manipulators with parametric uncertainty has been presented. The suitable performance of the control scheme is supported by the stability analysis in the Lyapunov sense and its efficiency was verified through implementations on a 2-DOF robot manipulator.
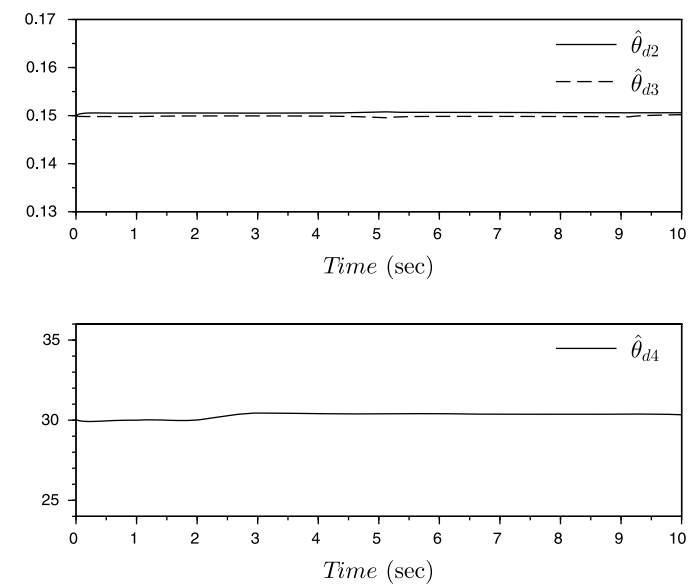

Fig. 8. Test 2: time evolution of the estimates of dynamic parameters.
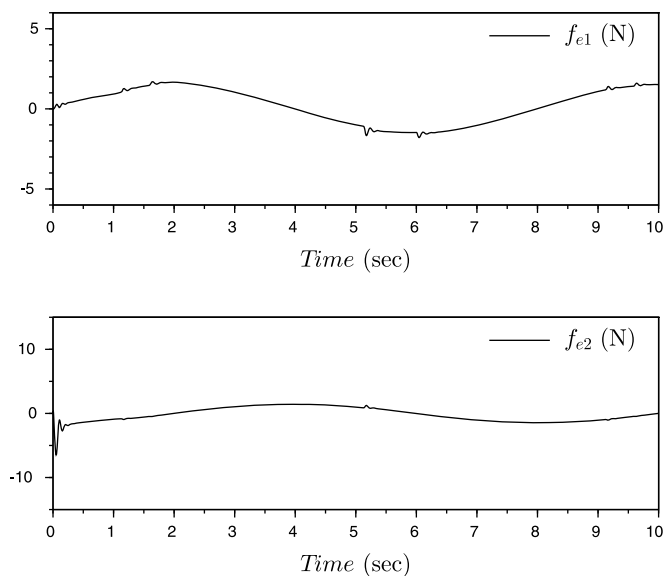

Fig. 9. Test 2: components of the interaction force.

The control scheme has a PD-like structure, where the generalized form of the proportional term gives rise to multiple particular controllers, permitting further innovation in their design and a wide range of possibilities for performance improvement.

By means of the two types of tests carried out, it is possible to verify the utility of the adaptive controller for trajectory tracking in both free and constrained spaces. Therefore, this control scheme can be used in applications such as robot assisted rehabilitation, where both passive (without the patient exerting force) and active (when the patient exerts force) movements are required during the development of therapy sessions.

One of the aspects to be improved, in future work, is the dependence of measurements of joint acceleration. In practice, this problem is solved by making use of filters that have as input the joint velocity. It is important to perform an experimental validation of the control scheme, as well as to improve the mathematical structure of the controller so that this practical solution be supported by a 
formal analysis of stability or robustness of the scheme.

\section{Acknowledgment}

The authors wish to thank CONACYT for the support provided through the project no. 222316.

\section{References}

Anderson, R.J. and Spong, M.W. (1988). Hybrid impedance control of robotic manipulators, IEEE Journal on Robotics and Automation 4(5): 549-556.

Belter, D., Łabecki, P., Fankhauser, P. and Siegwart, R. (2016). RGB-D terrain perception and dense mapping for legged robots, International Journal of Applied Mathematics and Computer Science 26(1): 81-97, DOI: 10.1515/amcs-2016-0006.

Canudas, C., Siciliano, B. and Bastin, G. (1996). Theory of Robot Control, Springer, London.

Carelli, R. and Kelly, R. (1991). An adaptive impedance/force controller for robot manipulators, IEEE Transactions on Automatic Control 36(8): 967-971.

Chiaverini, S., Siciliano, B. and Villani, L. (1999). A survey of robot interaction control schemes with experimental comparison, IEEE/ASME Transactions on Mechatronics 4(3): 273-285.

Chien, M.-C. and Huang, A.-C. (2004). Adaptive impedance control of robot manipulators based on function approximation technique, Robotica 22(4): 395-403.

Dulęba, I. and Opałka, M. (2013). A comparison of jacobian-based methods of inverse kinematics for serial robot manipulators, International Journal of Applied Mathematics and Computer Science 23(2): 373-382, DOI: 10.2478/amcs-2013-0028.

Gribovskaya, E., Kheddar, A. and Billard, A. (2011). Motion learning and adaptive impedance for robot control during physical interaction with humans, Proceedings of the 2011 IEEE International Conference on Robotics and Automation (ICRA), Shanghai, China, pp. 4326-4332.

Hagn, U., Nickl, M., Jörg, S., Passig, G., Bahls, T., Nothhelfer, A., Hacker, F., Le-Tien, L., Albu-Schäffer, A. et al. (2008). The DLR MIRO: A versatile lightweight robot for surgical applications, Industrial Robot: An International Journal 35(4): 324-336.

Haninger, K., Lu, J. and Tomizuka, M. (2016). Robust impedance control with applications to a series-elastic actuated system, Proceedings of the IEEE/RSJ International Conference on Intelligent Robots and Systems (IROS), Daejeon, South Korea, pp. 5367-5372.

He, W., Dong, Y. and Sun, C. (2016). Adaptive neural impedance control of a robotic manipulator with input saturation, IEEE Transactions on Systems, Man, and Cybernetics: Systems 46(3): 334-344.

Hogan, N. (1985). Impedance control: An approach to manipulation: Part I-Theory, Part II-Implementation, Part III-Applications, ASME Journal of Dynamic Systems, Measurement, and Control 107(1): 1-24.
Horn, R.A. and Johnson, C.R. (2012). Matrix Analysis, Cambridge University Press, New York, NY.

Hussain, S., Xie, S.Q. and Jamwal, P.K. (2013). Adaptive impedance control of a robotic orthosis for gait rehabilitation, IEEE Transactions on Cybernetics 43(3): 1025-1034.

Jianbin, H., Zongwu, X., Minghe, J., Zainan, J. and Hong, L. (2009). Adaptive impedance-controlled manipulator based on collision detection, Chinese Journal of Aeronautics 22(1): 105-112.

Jiang, Z.-H. (2005). Impedance control of flexible robot arms with parametric uncertainties, Journal of Intelligent and Robotic Systems 42(2): 113-133.

Kang, S.H., Jin, M. and Chang, P.H. (2009). A solution to the accuracy/robustness dilemma in impedance control, IEEE/ASME Transactions on Mechatronics 14(3): 282-294.

Kelly, R., Santibáñez, V. and Loría, A. (2005). Control of Robot Manipulators in Joint Space, Springer-Verlag, London.

Khalil, H.K. (1996). Nonlinear Systems, Prentice-Hall, Upper Saddle River, NJ.

Li, Z., Huang, Z., He, W. and Su, C.-Y. (2017). Adaptive impedance control for an upper limb robotic exoskeleton using biological signals, IEEE Transactions on Industrial Electronics 64(2): 1664-1674.

Lu, W.-S. and Meng, Q.-H. (1991). Impedance control with adaptation for robotic manipulations, IEEE Transactions on Robotics and Automation 7(3): 408-415.

Marchal-Crespo, L. and Reinkensmeyer, D.J. (2009). Review of control strategies for robotic movement training after neurologic injury, Journal of Neuroengineering and Rehabilitation 6(20): 1-15.

Martínez, P.A., Castelán, M. and Arechavaleta, G. (2016). Vision based persistent localization of a humanoid robot for locomotion tasks, International Journal of Applied Mathematics and Computer Science 26(3): 669-682, DOI: 10.1515/amcs-2016-0046.

Mendoza, M., Bonilla, I., Reyes, F. and González-Galván, E. (2012). A Lyapunov-based design tool of impedance controllers for robot manipulators, Kybernetika 48(6): 1136-1155.

Michel, A.N., Hou, L. and Liu, D. (2008). Stability of Dynamical Systems, Birkhaüser, Boston, MA.

Pérez-Ibarra, J.C., Dos Santos, W.M., Krebs, H.I. and Siqueira, A.A. (2014). Adaptive impedance control for robot-aided rehabilitation of ankle movements, Proceedings of the 5th IEEE RAS \& EMBS International Conference on Biomedical Robotics and Biomechatronics, Sao Paulo, Brazil, pp. 664-669.

Rahimifard, S., Talebi, H. and Mohammadi, A.D. (2016). Impedance control of non-passive bilateral teleoperation systems with uncertain dynamics, Proceedings of the 24th Iranian Conference on Electrical Engineering (ICEE), Shiraz, Iran, pp. 1931-1936. 
Reyes, F. and Kelly, R. (1997). Experimental evaluation of identification schemes on a direct drive robot, Robotica 15(05): 563-571.

Rodríguez-Liñán, M.C., Mendoza, M., Bonilla, I. and Chávez-Olivares, C.A. (2017). Saturating stiffness control of robot manipulators with bounded inputs, International Journal of Applied Mathematics and Computer Science 27(1): 79-90, DOI: 10.1515/amcs-2017-0006.

Rouche, N., Habets, P. and Laloy, M. (1977). Stability Theory by Lyapunov's Direct Method, Springer, New York, NY.

Sciavicco, L. and Siciliano, B. (2000). Modelling and Control of Robot Manipulators, Springer, London.

Sharifi, M., Behzadipour, S. and Vossoughi, G. (2012). Model reference adaptive impedance control of rehabilitation robots in operational space, Proceedings of the 4th IEEE $R A S$ \& EMBS International Conference on Biomedical Robotics and Biomechatronics (BioRob), Rome, Italy, pp. 1698-1703.

Sharifi, M., Behzadipour, S. and Vossoughi, G. (2014). Nonlinear model reference adaptive impedance control for human-robot interactions, Control Engineering Practice 32(1): 9-27.

Slotine, J.-J.E., Li, W. (1991). Applied Nonlinear Control, Prentice-Hall, Englewood Cliffs, NJ.

Song, A., Pan, L., Xu, G. and Li, H. (2015). Adaptive motion control of arm rehabilitation robot based on impedance identification, Robotica 33(9): 1795-1812.

Spong, M., Hutchinson, S. and Vidyasagar, M. (2005). Robot Modeling and Control, Wiley, New York, NY.

Takegaki, M. and Arimoto, S. (1981). A new feedback method for dynamic control of manipulators, ASME Journal of Dynamic Systems, Measurement, and Control 103(2): 119-125.

Wang, H. and Xie, Y. (2009). Adaptive inverse dynamics control of robots with uncertain kinematics and dynamics, Automatica 45(9): 2114-2119.

Xu, G., Song, A. and Li, H. (2011). Adaptive impedance control for upper-limb rehabilitation robot using evolutionary dynamic recurrent fuzzy neural network, Journal of Intelligent \& Robotic Systems 62(3): 501-525.

Yang, C., Chen, J. and Cheng, L. (2016). Neural learning enhanced teleoperation control of robots with uncertainties, Proceedings of the 9th International Conference on Human System Interactions (HSI), Portsmouth, $U K$, pp. 223-228.

Yarza, A., Santibanez, V. and Moreno-Valenzuela, J. (2013). An adaptive output feedback motion tracking controller for robot manipulators: Uniform global asymptotic stability and experimentation, International Journal of Applied Mathematics and Computer Science 23(3): 599-611, DOI: 10.2478/amcs-2013-0045.

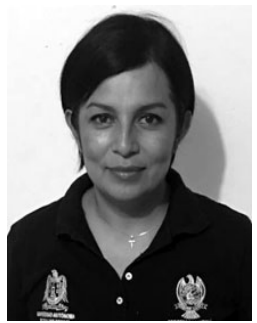

Isela Bonilla received the $\mathrm{BE}$ degree in communications and electronics engineering from the University of Colima, Mexico, in 2003, the MSc degree in electronics from the Autonomous University of Puebla, Mexico, in 2006, and the $\mathrm{PhD}$ degree in electrical engineering from the Autonomous University of San Luis Potosí in 2011. She joined the Autonomous University of San Luis Potosí, Mexico, in 2012, where she is presently a professor of electronic engineering and instrumentation. Her current research interests include impedance/force control and rehabilitation robotics.

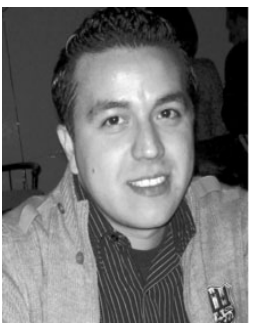

Marco Mendoza received the BE degree in communications and electronics engineering from the University of Colima, Mexico, in 2003, the MSc degree in electronics from the Autonomous University of Puebla, Mexico, in 2006, and the PhD degree in electrical engineering from the $\mathrm{Au}$ tonomous University of San Luis Potosí, Mexico, in 2011. He was an associate professor a the University of Sonora, Mexico, from 2011 to 2012. He joined the Autonomous University of San Luis Potosí, Mexico, in 2014, where he is presently a professor of biomedical engineering. His current research interests include robot control and biorobotics.

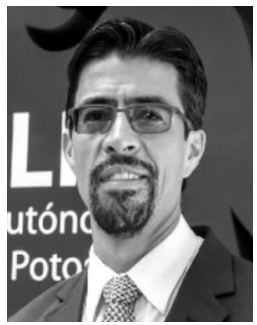

Daniel U. Campos-Delgado received the BS degree in electronics engineering from the Autonomous University of San Luis Potosí, Mexico, in 1996, and the MSEE and PhD degrees in electrical engineering from Louisiana State University (LSU), Baton Rouge, LA, USA, in 1999 and 2001, respectively. In 2001, he joined the College of Sciences of the Autonomous University of San Luis Potosí as a professor. He has published more than 170 referred papers in scientific journals and congresses. His research interests include estimation and control algorithms, optimization, dynamic modeling, and optima signal processing. Prof. Campos-Delgado is currently a member of the Mexican Academy of Sciences and a senior member of the Institute of Electrical and Electronics Engineers (IEEE).

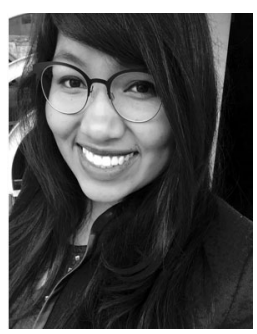

Diana E. Hernández-Alfaro received the BS and $\mathrm{MSc}$ degrees in electronics engineering from the Autonomous University of San Luis Potosí, Mexico, in 2011 and 2013, respectively. She received the Best Master's Thesis award from the Mexican Robotics Association in 2014. She joined Ericsson in 2014 as an engineer, where she is currently working on telecommunications with LTE, UMTS and GSM technologies in various configurations.
Received: 29 March 2017

Revised: 6 October 2017

Re-revised: 23 November 2017

Accepted: 13 December 2017 